Sonderdruck aus: „Zeitschrift für Kristallographie“, 136 (1972) 350-386

Herausgegeben von G. E. Bacon, M. J. Buerger, F. Laves, G. Menzer, I. N. Stranski

\title{
A new method to measure general birefringence in crystals
}

\author{
By R. VERREAULT* \\ Laboratorium für Festkörperphysik, E.T.H., Zürich
}

(Received 5 July 1971)

AKA DEM I CHE VERLAGSGESELLSCHAFT

FRA N K U R T A M A N

1972 


\section{Auszug}

Die Streuungseigenschaften eines anisotropen festen Körpers ermöglichen die Beobachtung und die Messung von linearer, zirkularer und elliptischer Doppelbrechung. Eine allgemeine Theorie dieser Erscheinung für jede Art von Doppelbrechung und für jeden Polarisationszustand der einfallenden Lichtschwingung wird gegeben. Betrachtet werden streuende Teilchen sowohl mit Querschnittdimensionen $a \ll \lambda$ (Rayleighscher Grenzfall) als auch mit $a \approx \lambda$. Aus der Theorie ergibt sich, daß die Streuzentrenverteilung einer quasi-kontinuierlichen Analysator-Verteilung, die eine simultane Analyse des durchgehenden Lichtbündels auf seinem ganzen Weg durch das (im allgemeinen elliptisch) doppelbrechende Medium bewirkt, äquivalent ist. Ein neues Verfahren an Hand von Oberflächenstreuung wird beschrieben. Es ermöglicht, visuelle Beobachtungen an sehr perfekten (also wenig streuenden) Kristallen mit Hilfe schwacher Lichtquellen durchzuführen, ohne daß es nötig wäre, für vollkommene Dunkelheit in der Umgebung der Probe zu sorgen. Die Oberflächenstreuungsmethode ist für Präzisionsmessungen besonders gut geeignet; sie wird an Hand der elliptischen Doppelbrechung von Quarz gründlich geprüft. Beide Methoden von Oberflächen- und Raumstreuung werden auf verschiedene Arten der Doppelbrechung in Einkristallen von $\mathrm{Eu}_{2} \mathrm{SiO}_{4}$ und EuTe angewendet.

\section{Résumé}

Les propriétés diffusantes d'un solide anisotrope permettent l'observation et la mesure de la biréfringence linéaire, circulaire et elliptique. Nous analysons le phénomène d'une façon générale en tenant compte de chaque type de biréfringence, de tous les états de polarisation de la vibration lumineuse incidente et des dimensions des centres diffusants aussi bien dans le domaine $a \ll \lambda$ (limite de Rayleigh) que $a \approx \lambda$. Comme conséquence de la théorie, la distribution des centres diffusants est équivalente à une répartition quasi-continue d'analyseurs fournissant une analyse simultanée du faisceau transmis sur toute sa trajectoire dans le milieu (en général elliptiquement) biréfringent. Nous décrivons une nouvelle technique basée sur la diffusion en surface. Elle permet de procéder à des observations visuelles sur des cristaux de haute perfection, donc

* Now at Université du Québec, Chicoutimi, Québec, Canada. 
diffusant faiblement, tout en utilisant des sources lumineuses à faible puissance et sans qu'il soit nécessaire de réaliser l'obscurité complète autour de l'échantillon. La méthode de la diffusion en surface se prête particulièrement bien aux mesures de précision; nous en faisons une vérification soignée au moyen de la biréfringence elliptique du quartz. Les divers types de biréfringence rencontrés dans des monocristaux de $\mathrm{Eu}_{2} \mathrm{SiO}_{4}$ et EuTe font ensuite l'objet d'une application des deux méthodes, à savoir diffusion en surface et diffusion en profondeur.

\section{Abstract}

The scattering properties of an anisotropic solid for polarized light allow the observation and the measurement of linear, circular and elliptic birefringence. A general analysis of the phenomenon for any kind of birefringence and any state of polarization of the incident light vibration is given. Scattering by particles with size $a \ll \lambda$ (Rayleigh limit) or $a \approx \lambda$ is considered. It follows from the theory that the scattering-center distribution is equivalent to a quasi-continuous array of analyzers providing a simultaneous analyzation of the transmitted beam along its whole path in the (in general elliptically) birefringent medium. A new technique based on surface scattering is described. It allows visual observations to be made on very perfect (hence weakly scattering) crystals with lowpower light sources and without being forced to achieve complete darkness in the surroundings of the sample. The method of surface scattering is especially adequate for precision measurements; it is thoroughly tested with the elliptic birefringence of quartz. Both methods of surface and bulk scattering are applied to various kinds of birefringence in single crystals of $\mathrm{Eu}_{2} \mathrm{SiO}_{4}$ and EuTe.

\section{Introduction}

In an optical investigation of anisotropic crystals, birefringence is one of the first properties which have to be considered. The natural birefringence of a substance provides an important identification criterion in petrographic work, while in physical studies like piezo-, electroor magneto-optics, a quantitative knowledge of both the natural and the induced kinds of birefringence is essential. In its most general form, birefringence is defined as the difference in the refractive indices of an anisotropic medium for the two orthogonally, in general elliptically, polarized light vibrations which are transmitted unchanged along a given direction of propagation (the notion of orthogonality will be clarified below). Linear birefringence and circular birefringence are special cases of the above one, where the orthogonal vibrations are, respectively, two linearly polarized ones with their azimuths differing by $90^{\circ}$, or two left and right circularly polarized ones. Since elliptic birefringence can be thought of as resulting in a unique way from the simultaneous presence of linear and circular birefringence, the last two are the ones most frequently dealt with. When not otherwise specified, birefringence will be meant in its general sense throughont this paper. 
The usual methods of measuring birefringence can be classified into two categories:

a) the differential or direct methods, according to which one measures the phase retardation or the optical path difference between the orthogonal vibrations propagated without change in an anisotropic medium;

b) the absolute or indirect methods, according to which one measures the principal refractive indices individually and then takes their difference.

Since the method described in this paper belongs to the first category, we shall endeavour to compare it only with direct methods. A description of all the methods available for the direct measurement of birefringence is beyond the scope of this article. This can be found in standard textbooks on petrography or on optical crystallography ${ }^{1-4}$. Suffice it to say that they all involve the process of analyzing the light transmitted by the sample with instruments such as polarizing microscopes, polarimeters, ellipsometers, etc.

The widely used technique of polarization microscopy in connection with platelet-like crystals observed in transmission is practically limited to thin samples, because of the fact that the differences between the dispersion of the sample and that of the compensator material make the unique determination of the order of the measured path difference almost impossible except for the very first orders. On the other hand, the alternative method of tracking the path difference up to high orders along the varying thickness of a wedge with sufficiently thin edge is often handicaped by the lapping difficulties encountered in a great many substances, which are brittle or cleave into layers or otherwise form twins easily. In contrast with that situation, two of the main characteristics of the light-scattering configuration for measuring birefringence are the following:

a) The beam of transmitted light is observed from the side and the order of the path difference can be directly counted along the direction

1 A. Johannsen, Manual of petrographic methods. McGraw-Hill Book Co., New York, 1918.

2 E. E. WAHLSTROM, Optical crystallography. John Wiley \& Sons, Inc., New York, $4^{\text {th }}$ ed., 1969.

3 G. N. Ramachandran and S. Ramaseshan, Crystal optics. Handb. Physik (FLÜGGE) XXV/I, Springer, Berlin 1961, pp. $1 \mathrm{ff}$.

4 R. RAtH, Kristallographie. Philips Technische Bibliothek. Eindhoven. Niederlande, 1965, Chap. II. 
of propagation. For an adequately monochromatic source, the limit to the uniquely determined order of the path difference is then set by the available dimensions of the crystal or by its perfection.

b) While a quantitative measurement of birefringence is practically possible only for one direction of propagation in a platelet or in a wedge, the light-scattering configuration allows quantitative measurements to be made through many faces of a single appropriately shaped bulky specimen, and in each face, for a wide continuous range of directly observed directions of propagation within the bulk.

Although the light-scattering configuration can be applied to crystals of microscopic dimensions, the above characteristics make it more properly suited to large specimens. The light-scattering configuration for measuring birefringence can therefore be considered as a method which is complementary to the traditional ones.

Historically, the observation of optical activity from the light scattered in a solid goes back to 1919 , as Lord RAYLEIGH ${ }^{5}$ could detect the trace of a beam of sunlight in a piece of smoky quartz which was mounted in a specially designed observation cell in order to ensure the total darkness of the background. The same phenomenon has been studied by CHANDRASEKHARAN ${ }^{6}$ in 1947 and used to determine the selection rules pertaining to the Raman scattering of quartz for light propagation along the optic axis 7 . The light-scattering configuration was extended by him ${ }^{8}$ to the case of linear birefringence with the stress birefringence of plexiglass and bakelite. Only the Rayleigh scattering of a linear incident vibration in the bulk of the sample was considered, so that the $\lambda^{-4}$ dependence of the scattered intensity limited the observations to the technique of long-exposure photography mostly in the ultraviolet spectral region.

In this paper, a general analysis of the phenomenon for any kind of birefringence and any state of polarization of the incident vibration is given. The scattering by particles comparable in size with the wavelength of the radiation used is also included. As suggested by RAY-

5 R. J. Strutw, Scattering of light by solid substances. Proc. Roy. Soc. [London] A 95 (1919) $476-479$.

6 V. Chandrasekharan, The scattering of light in quartz. Proc. Ind. Acad. Sci. A 25 (1947) 256-259.

7 V. ChandRasekharan, The influence of optical activity on light-scattering in quartz. Proc. Ind. Acad. Sci. A 28 (1948) 409-416.

$8 \mathrm{~V}$. Chandrasekharan, The scattering of polarized light beams in birefringent solids. Proc. Ind. Acad. Sci. A 26 (1947) 110-113. 
LEIGH $^{5}$ for the case of optical activity, it follows from the theory that the scattering-center distribution is equivalent to a quasi-continuous array of analyzers providing a simultaneous analyzation of the transmitted beam along its whole path in the (in general elliptically) birefringent medium. A new technique based on surface scattering is described. It allows visual observations to be made on very perfect crystals with ordinary low-power light sources and without being forced to achieve complete darkness in the surroundings of the sample. The method of surface scattering is especially adequate for precision measurements. It is thoroughly tested hereafter with the elliptic birefringence of quartz. Both methods of surface and bulk scattering are then applied to various kinds of birefringence in single crystals of $\mathrm{Eu}_{2} \mathrm{SiO}_{4}$ and EuTe.

\section{Theoretical background}

\section{A. The Poincaré representation}

The analysis of the phenomena described hereafter will be made in terms of the Poincare representation of polarized light ${ }^{9}, 3(p .1)$. The principles of the representation are shortly recalled, together with a definition of the nomenclature used.

\section{Definitions}

The state of polarization of an electromagnetic wave is generally represented by the elliptical trace of the $D$ vector in a plane $(x y)$ perpendicular to the wave normal (or direction of propagation) $\mathrm{Oz}$ (Fig. $1 \mathrm{a}$ ). The major axis $2 a$ of the ellipse lies at an azimuth $\psi$ with respect to the $x$ axis, the ellipticity is characterized by an angle $\omega$ such that the axial ratio $b / a=\tan \omega$, and finally the $D$ vector can describe the ellipse in a clockwise (right-handed ellipse) or counter-clockwise (left-handed ellipse) motion for an observer looking towards the source. Alternatively, the same state can be represented by a point $P$ on the surface of a sphere (Poincaré sphere) with a longitude $2 \psi$ and a latitude $\pm 2 \omega$, the $(+)$ and $(-)$ signs corresponding respectively to left-handed and right-handed ellipses (Fig. $1 \mathrm{~b}$ ). In the limiting case $\omega=0$, one has linearly polarized light, represented by points on the equator. The $x$ and $y$ axes, in particular, can be identified with two states of linear polarization and hence be represented by the diametrically opposed

${ }^{9} \mathrm{H}$. PoInCaré, Theorie mathématique de la lumière, Vol. II, Chap. XII, Paris 1892. 
a)

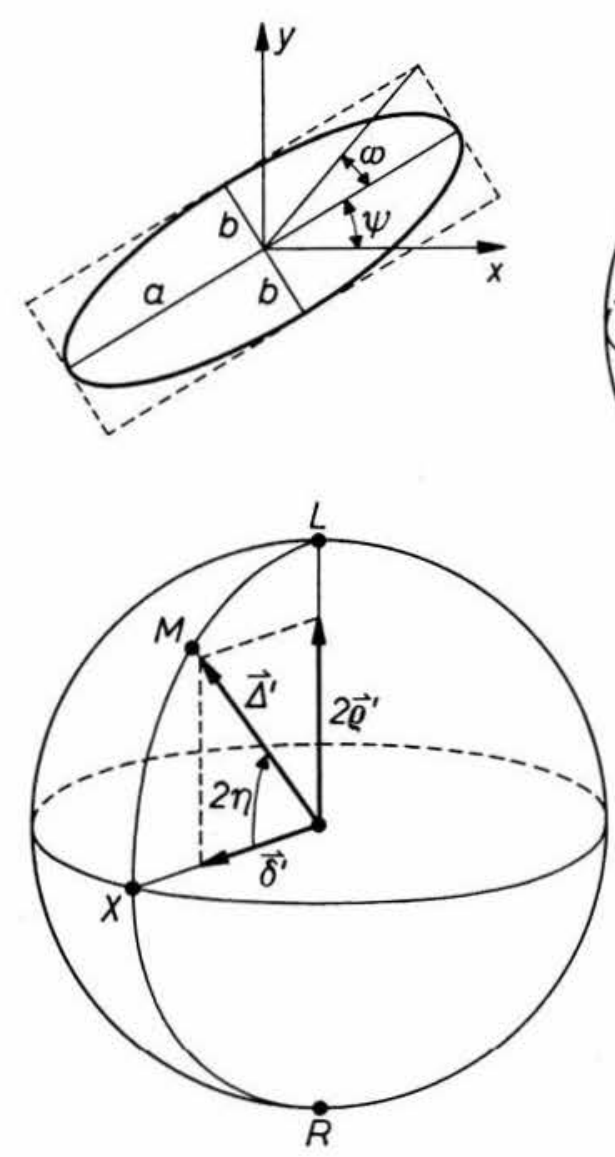

c) b)
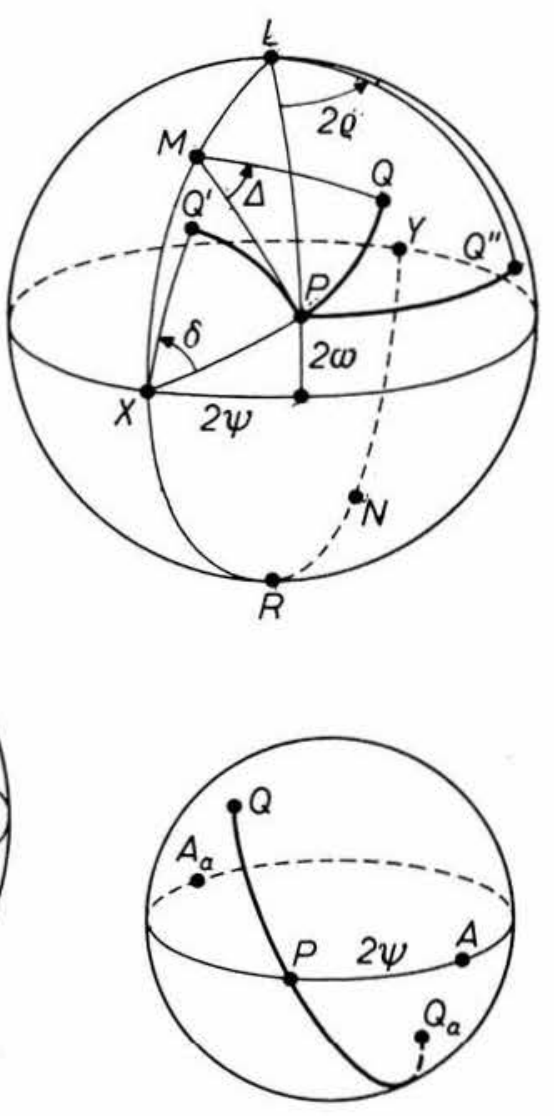

d)

Fig.1. Representations of polarized light in birefringent media: a) elliptical trace of the $D$ vector; b) Poincaré sphere: effect of a birefringent medium on a polarized beam; c) composition of linear and circular birefringence to yield elliptical birefringence; d) decomposition of a polarization state $P$ into any two orthogonal components $\left(Q\right.$ and $\left.Q_{\mathrm{a}}\right)$ or into the states transmitted $(A)$ and extinguished $\left(A_{\mathrm{a}}\right)$ by a linear analyzer

points $X$ and $Y$ on the equator. At the other limits of $\omega( \pm \pi / 4)$, one has left and right circularly polarized vibrations, represented by the north and south poles $L$ and $R$ respectively. For the sake of brevity, we shall often make use of the metonymical expressions "vibration $P$ " or "state $P$ " hereafter, to speak of a vibration in the state of polarization represented by the point $P$ on the Poincaré sphere.

Two vibrations are said to be orthogonal when their representing ellipses have: a) the same ellipticity; b) their major axes at right angle to each other; c) opposite hands. It can be verified that two orthogonal vibrations are represented by two antipodal points on the Poincaré sphere. 
The orientation of a birefringent plate can uniquely be determined, except for the degenerate case of circular birefringence, by the two states of polarized light which it transmits unchanged. If no dichroism is present, those states $M$ and $N$ (Fig. 1b) are orthogonal and they define a diametral axis of rotation of the Poincaré sphere. For brevity, the expression "plate $M N$ " will be used in the following, its meaning being now obvious.

Any polarized vibration can be uniquely resolved into two components along the orthogonal states $M$ and $N$. For a vibration $P$ incident on the birefringent plate $M N$, the state $Q$ of the transmitted vibration is obtained by rotating the Poincaré sphere about the diameter $M N$ through an angle $\Delta$ equal to the phase retardation introduced by the plate between the $N$ and $M$ components of the vibration $P$. The rotation of the spherical surface is counter-clockwise about the point representing the faster state (here $M$ ), for an observer looking towards the center of the sphere. In the particular case of a linearly birefringent plate $X Y$, the axis of rotation lies in the equatorial plane and the incident vibration is resolved into two linearly polarized ones. The direction of vibration of the states $X$ and $Y$ are respectively called the fast and slow axes of the plate and the phase retardation introduced by the latter between the linear components of the incident vibration along those axes is denoted by $\delta$. This yields the transmitted vibration $Q^{\prime}$ in Fig. $1 \mathrm{~b}$. When a transparent plate shows only circular birefringence (e.g. optical activity and/or one-way Faraday rotation), the axis of rotation of the Poincaré sphere is the polar axis $L R$ and the phase retardation between the two circularly polarized components into which the incident vibration $P$, in general elliptic, is then resolved amounts to $2 \varrho$. The resulting transmitted vibration $Q^{\prime \prime}$ has the same ellipticity as $P$ but the major axis of its representing ellipse has undergone the azimuthal rotation $\varrho$.

As mentioned before, the general case of elliptic birefringence arises from the simultaneous presence of linear and circular birefringence. An elliptically birefringent plate can actually be thought of as a pile of alternate linearly $(X Y)$ and circularly $(L R)$ birefringent plates of equal vanishing thicknesses and with the same orientation for all the plates $X Y$. The respective phase retardations per unit thickness in each kind of plate are $\delta^{\prime}$ and $2 \varrho^{\prime}$. From the commutativity of the infinitesimal rotations of a sphere about different axes, both kinds of plate can be paired in any order, each pair yielding by vectorial composition of the rotations of the Poincaré sphere a unique resultant phase 
retardation per unit thickness (Fig. 1 c)

$$
\vec{\Delta}^{\prime}=\vec{\delta}^{\prime}+2 \vec{\varrho}^{\prime} .
$$

The elliptic states $M$ and $N$ propagated unchanged through the finite elliptically birefringent plate are such that the major axes of the representing ellipses are respectively along the fast and slow axes $X$ and $Y$ of the composing infinitesimal plates with pure linear birefringence, and their ellipticity is characterized by the latitude $2 \eta$, where

$$
\tan 2 \eta=\frac{2 \varrho^{\prime}}{\delta^{\prime}} \text {. }
$$

The axes $X$ and $Y$ are called principal directions of the elliptically birefringent plate $M N$. The magnitude of the composite phase retardation $\Delta$ introduced by the plate $M N$ of finite thickness $t$ between the $N$ and $M$ components of the incident vibration is given by

$$
\Delta=t \Delta^{\prime}=t\left[\left(\delta^{\prime}\right)^{2}+\left(2 \varrho^{\prime}\right)^{2}\right]^{1 / 2} .
$$

\section{Intensity transmitted by an analyzer}

The intensity $I_{Q}$ of the component of any vibration $P$ of intensity $I_{P}$ along an arbitrary polarization state $Q$ (Fig. $1 \mathrm{~d}$ ) is given by

$$
I_{Q}=I_{P} \cos ^{2} \frac{1}{2}(P Q),
$$

and along the state $Q_{\mathrm{a}}$ orthogonal (i.e. antipodal) to $Q$, by

$$
I_{Q_{\mathrm{a}}}=I_{P} \cos ^{2} \frac{1}{2}\left(P Q_{\mathrm{a}}\right)=I_{P} \sin ^{2} \frac{1}{2}(P Q),
$$

where $P Q$ and $P Q_{\mathrm{a}}$ are great circular arcs on the Poincaré sphere. A linear analyzer (nicol, polaroid, etc.) can be thought of as a linearly birefringent plate with infinitely large linear dichroism. Any vibration $P$, in general elliptic, incident on it is resolved along the fast and slow axes $A$ and $A_{\mathrm{a}}$ of that plate and only one of those components, say $A$, is transmitted, with an intensity

$$
I_{A}=I_{P} \cos ^{2} \frac{1}{2}(P A)=I_{P} \sin ^{2} \frac{1}{2}\left(P A_{\mathrm{a}}\right) .
$$

The last member gives the transmitted intensity in terms of the extinction direction of the analyzer. In the special case (Fig. 1d) where $P$ represents a linearly polarized vibration at an azimuth $\psi$ with respect to the transmission direction $A, P A=2 \psi$, and one has the well-known 
cosinus-squared dependence of the transmitted intensity on the azimuth of the analyzed vibration:

$$
I_{A}=I_{P} \cos ^{2} \psi .
$$

For crossed polarizer and analyzer, $P$ and $A_{\mathrm{a}}$ coincide, whence $I_{\boldsymbol{A}}=0$.

B. Analyzer equivalent of a scattering center in the Rayleigh limit

In contrast with a pure gas or a pure liquid, an ideal crystal should show no scattering at all. Real crystals, however, show not only the inelastic light scattering of the Brillouin and Raman types caused by phonons, but also observable amounts of elastic scattering due to random fluctuations in stoichiometry, to inclusions, to dislocations, to domains of ferroelectric or of magnetic nature, etc. Detailed treatments of some simplified cases can be found in the literature ${ }^{10-13}$. For the purpose of this analysis, though, a merely qualitative knowledge of the intensity pattern and of the polarization features of the light scattered in a few important planes is required. The limiting case of Rayleigh scattering, where the average cross-dimension $a$ of the scattering centers is much smaller than the wavelength of the radiation used $(a \ll \lambda)$, will first be considered in this section. As shown hereafter, the properties of interest for this study can be described by a particularly simple analyzer model.

\section{Rayleigh scattering of a linear vibration}

The Rayleigh scattering of a linearly polarized vibration is described by the far-field radiation originating from crossed electric and magnetic vibrating dipoles. Let

a) the plane of observation be the plane containing the directions of propagation of both the directly transmitted beam and the observed scattered beam;

10 H. Hönl, A. W. Maue und K. Westpfahl, Theorie der Beugung. Handb. Physik (FLÜGGE) XXV/I, Springer, Berlin 1961, pp. $218 \mathrm{ff.}$

11 H. C. VAN DE Hulst, Light scattering by small particles. Wiley, New York 1957.

12 M. Born and E. Wolf, Principles of optics. Pergamon Press, London 1959.

13 M. A. Schirmann, Dispersion und Polychroismus des polarisierten Lichtes, das von Einzelteilchen von der Größenordnung der Wellenlänge des Lichtes abgebeugt wird. Ann. Physik [4] 59 (1919) 493-537. 
b) $\theta$ be the scattering angle, i.e. the angle between the above directions of propagation, measured in such a way that $0<\theta<\pi / 2$ corresponds to forward scattering and $\pi / 2<\theta<\pi$ to backward scattering;

c) $\psi$ be the azimuth of the plane of vibration of the transmitted beam with respect to the plane of observation.

While the light scattered at $\theta \neq \pi / 2$ has vibrating components in and normal to the plane of observation, the $90^{\circ}$-Rayleigh scattering is characterized by the fact that the in-plane component of vibration is zero for every value of $\psi$. In this particular case, both the direction of propagation and the direction of vibration of the scattered light are in a plane normal to the transmitted beam and hence can be identified with two possible orthogonal states of linear polarization of the latter, say $S$ and $S_{\text {a }}$ respectively. It becomes therefore possible to represent the observed scattered beam at $\theta=\pi / 2$ by two equatorial points $S$ and $S_{\mathrm{a}}$ on the Poincaré sphere describing the polarization states of the directly transmitted beam.

For an incident linear vibration $T$ (Fig. 2) with an intensity $I_{T}$, the intensity of the scattered vibration propagated normally to the transmitted beam and at an angle $\psi$ with respect to the vibration plane of the latter is, from the electromagnetic theory of radiation,

$$
I=k I_{T} \sin ^{2} \psi,
$$

where $k$ is a factor of the form $a^{6} / \lambda^{4}$. Apart from the constant factor $k$, the azimuthal dependence of the scattered intensity is the same as that of the intensity which one would observe along the transmitted beam if the scattering center were replaced by a linear analyzer. The extinction direction $A_{\mathrm{a}}$ of the analyzer would then coincide with the propagation direction $S$ of the scattered vibration and the transmitted intensity would be

$$
I_{A}=I_{T} \sin ^{2} \frac{1}{2}\left(T A_{\mathrm{a}}\right)=I_{T} \sin ^{2} \frac{1}{2}(T S)=I_{T} \sin ^{2} \psi .
$$

Such an analyzer equivalence can account namely for the Rayleigh scattering in a medium showing only circular birefringence.

\section{Rayleigh scattering of an elliptic vibration}

In the case where the incident vibration $P$ is elliptically polarized, it can be resolved into any two orthogonal linear states $X$ and $Y$ (Fig. 2). The $X$ - and $Y$-components are coherent with a constant phase 


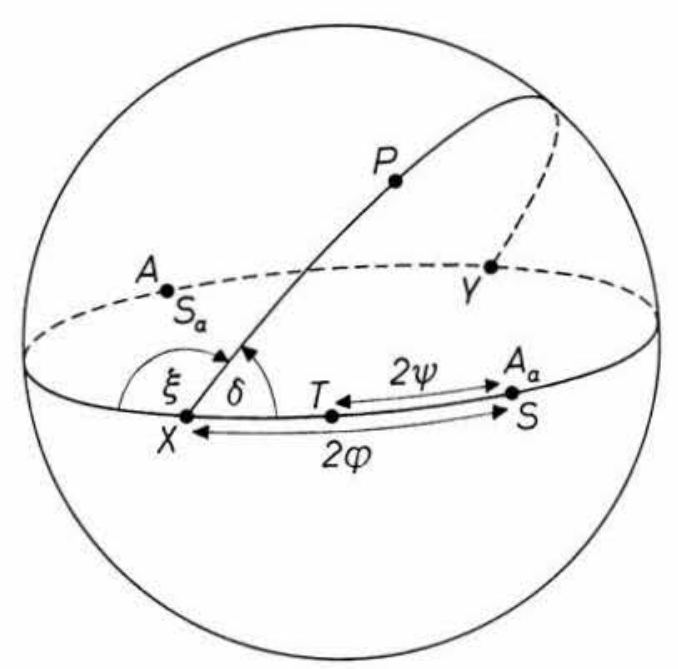

Fig. 2. Representation of the scattered beam in $90^{\circ}$ Rayleigh scattering on the Poincaré sphere pertaining to the polarization states of the directly transmitted beam

difference $\delta$ between them. Assuming each of those components to be scattered independently, the scattered contributions in a direction $S$ defined as above are resolved into the same linear state, namely that corresponding to $S_{\mathrm{a}}$, with the respective intensities

$$
\begin{aligned}
& I_{(X)}=k I_{X} \sin ^{2} \varphi, \\
& I_{(Y)}=k I_{Y} \cos ^{2} \varphi,
\end{aligned}
$$

where $\varphi$ is the azimuth of the plane of vibration of the $X$ component with respect to the plane of observation. Since the Rayleigh scattering is coherent, the two scattered contributions can then interfere. One can show $^{3}$ (p. $9 \mathrm{ff}$ ) that their phase difference is $(\delta-\xi)$, where $(-\xi)$ is an additional phase difference introduced by the process of taking components of the $X$ and $Y$ vibrations along a state $S_{\mathrm{a}}$ which does not lie on the great circular are $X P Y$. This are is namely the locus of the elliptic states obtained by composing $X$ - and $Y$-vibrations with various relative intensities but with a constant phase difference $\delta$. The observed scattered intensity is therefore obtained by coherently adding the intensities of both contributions, that is

$$
I=k\left\{I_{X} \sin ^{2} \varphi+I_{Y} \cos ^{2} \varphi+2\left[I_{X} I_{Y} \sin \varphi \cos \varphi \cos (\delta-\xi)\right]^{1 / 2}\right\} .
$$

Except for the factor $k$, this is precisely the intensity which a linear analyzer would transmit along the direction of the incident beam, if it were placed at the site of the scattering center with its extinction direction lying in the plane of observation. This situation accounts for the 
Rayleigh scattering occurring in generally birefringent media, apart from subsequent changes in the polarization state of the scattered beam, if the medium in which the latter travels also has birefringence for the propagation direction of the scattered wave under consideration. One can therefore make the statement that a distribution of scattering centers with $a \ll \lambda$ along a polarized beam in a birefringent medium is equivalent to a similar distribution of linear analyzers, in so far as the scattered intensity in a plane normal to the directly transmitted beam is concerned. The scattering pattern provides in this way a quasi-continuous simultaneous analyzation of the directly transmitted beam along its path in the birefringent medium.

C. Light-scattering configuration for the observation of birefringence

\section{General birefringence (normal incidence)}

Let a plane wave in a state $P_{0}$, in general elliptic (see Fig. 3 ), enter the plane face $z=0$ (entrance facet) of an elliptically birefringent plate $M N$ at normal incidence, the $z$ axis lying along the wave normal (direction of propagation) within the plate, and the $x$ and $y$ axes coinciding with the principal directions $X$ and $Y$ of the plate for that direction of propagation. The distribution of the states of polarization along the $z$ axis is such that the representing point $P(z)$ on the Poincare sphere describes in general a small circle in counter-clockwise motion centered on the faster state $M$ as $z$ increases. For a direction of observation $S$ in the $x y$ plane at an azimuth $\varphi$ with respect to the $x$ axis, the scattered intensity is

$$
I=k I_{0} \sin ^{2} \frac{1}{2}(P S),
$$

where the small attenuation of the transmitted light is neglected and $P S$ is the great circular arc joining $P(z)$ and $S$. The intensity is therefore a periodic function of $z$ with minima for $P$ at $E$ and maxima for $P$ at $F$ on the great circle $M S N$. There is a fringe system parallel to the entrance facet with the fringe width

$$
\Lambda=\frac{2 \pi}{\Delta^{\prime}}=2 \pi\left[\left(\delta^{\prime}\right)^{2}+\left(2 \varrho^{\prime}\right)^{2}\right]^{-1 / 2}
$$

and the positions of the dark fringes at

$$
z_{m}=\left(J+\frac{P_{0} E}{2 \pi}\right) \Lambda \quad(J=0,1,2, \ldots),
$$




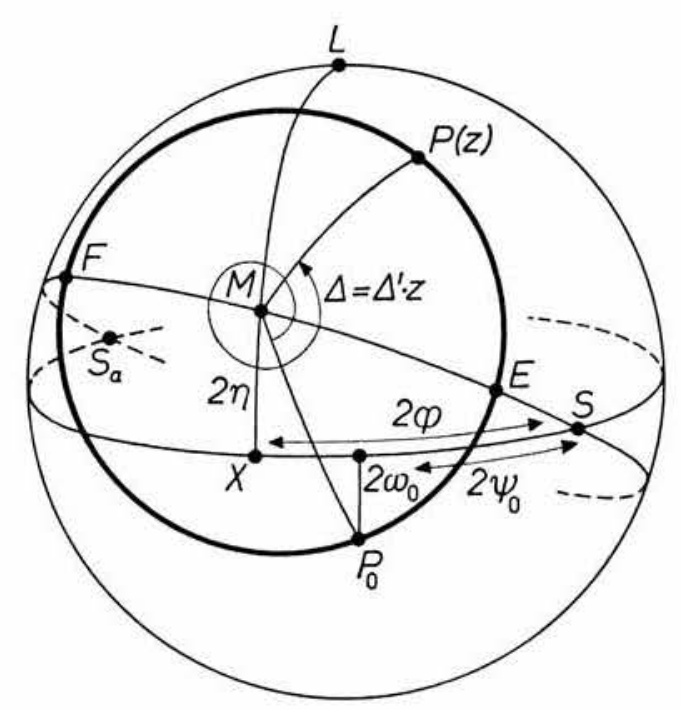

Fig. 3. Poincaré representation for the $90^{\circ}$ Rayleigh scattering in an ellipticallybirefringent medium

where the $\operatorname{arc} P_{0} E$ is measured along the circle (in general a small circle) described by $P(z)$, starting from $P_{0}$ in the direction of motion of $P(z)$.

The visibility of a fringe system is defined as

$$
V=\frac{I_{\mathrm{M}}-I_{\mathrm{m}}}{I_{\mathrm{M}}+I_{\mathrm{m}}}
$$

where $I_{\mathrm{M}}$ and $I_{\mathrm{m}}$ are respectively the maximal and minimal intensities. It is clear from Fig. 3 that the visibility will be maximum when $E$ and $S$ coincide, i.e. when the transmitted vibration at the site of a dark fringe is linearly polarized $\left[I_{\mathrm{m}}=k I_{0} \sin ^{2} \frac{1}{2}(E S)=0\right]$. Moreover, the average brightness of the scattering pattern will be maximum when $F$ and $S_{\mathrm{a}}$ coincide, i.e. when the transmitted vibration at the site of a bright fringe is linearly polarized at $90^{\circ}$ to the direction of observation. This is easily realized in practice by orienting the plate in such a way that both principal directions are at $45^{\circ}$ to the direction of observation $\left(S X=2 \varphi=90^{\circ}\right)$ and by using a linear incident vibration parallel to the direction of observation or perpendicular to it $\left(P_{0}=S\right.$, or $\left.P_{0}=S_{\mathrm{a}}\right)$.

The latter condition pertaining to the brightness of the scattering pattern has been improperly assigned by CHANDRASEKHARAN 8 to the visibility of the fringes. However, as illustrated below in the case of europium orthosilicate, a fringe system with optimum contrast can of course be obtained as soon as both the state $E$ and the direction of observation $S$ in Fig. 3 are distinct from a privileged vibration (in the general case, $M$ or $N$ ). The only necessary condition for the optimum fringe visibility is the coincidence of $E$ and $S$. 


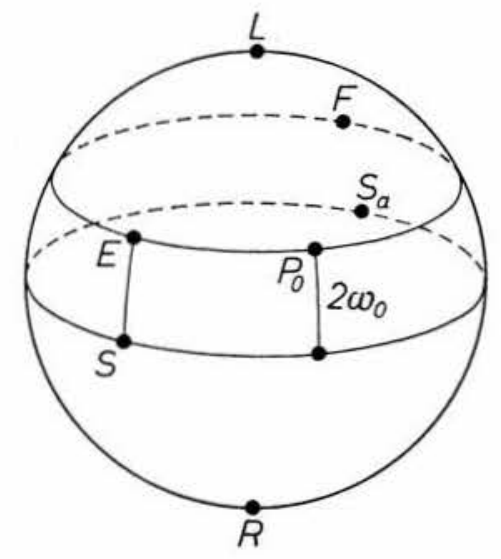

a)

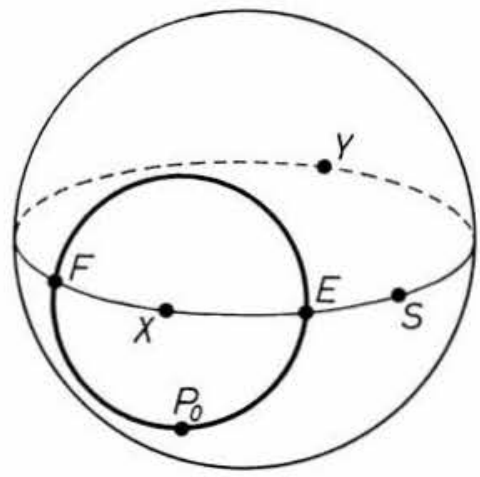

b)

Fig. 4. Poincaré representation for the $90^{\circ}$ Rayleigh scattering in a) a circularlybirefringent medium; b) a linearly-birefringent medium

\section{Circular limit}

In the case of a circularly birefringent plate, $2 \eta=\pi / 2$ in Fig. 3 and the locus of $P(z)$ on the Poincaré sphere is in general a small circle parallel to the equator which passes through $P_{0}$ (Fig. $4 \mathrm{a}$ ). The ellipticity of the transmitted state remains uniform throughout the plate $\left(b / a=\tan \omega_{0}\right)$ but the azimuth of the major axis of the ellipse rotates uniformly as $z$ increases. The minimal and maximal scattered intensities are then respectively

$$
\begin{aligned}
& I_{\mathrm{m}}=k I_{0} \sin ^{2} \omega_{0}, \\
& I_{\mathrm{M}}=k I_{0} \cos ^{2} \omega_{0} .
\end{aligned}
$$

Again, the brightness and the visibility of the fringe system are maximal when the incident vibration is linearly polarized. In contrast with the general case, the azimuth of $P_{0}$ has no influence on the visibility, since the arc $S E=2 \omega_{0}$ independently of $\psi_{0}$.

The hand of the circularly birefringent plate $L R$ can be determined by varying the azimuth of the incident vibration, which can be assumed linear, for simplicity. In a left-handed crystal, $L$ is the faster state and $P(z)$ moves then along the equator in the sense of increasing azimuth (counter-clockwise about $L$ ). By rotating the polarizer in the positive direction, $P_{0}$ is also displaced along the equator in the counter-clockwise sense about $L$ and the arc $P_{0} E$ in (2) decreases. This means that the first dark fringe gets closer to the entrance facet $z=0$, so that the whole fringe system is shifted towards the source. Correspondingly, a 
right-handed crystal is characterized by a shift of the fringe system away from the source on rotating the polarizer in the counter-clockwise (positive) sense, for an observer looking towards the source.

\section{Linear limit}

When the anisotropic plate shows only linear birefringence, one has $2 \eta=0$ in Fig. 3 , so that $P(z)$ describes a circle centered on the point $X$ (Fig. $4 \mathrm{~b}$ ). The transmitted beam is then linearly polarized at the sites where the scattered intensity reaches its extremal values and the optimum conditions of visibility and brightness are analogous to those of the general case.

The sign of the linear birefringence $\left(n_{Y}-n_{X}\right)$ and, in a principal section parallel to the axis of a uniaxial crystal, the optic sign of the crystal can be determined in a manner similar to that used for the hand of an optically active crystal, namely by displacing $P_{0}$ along the locus of $P(z)$ and by observing the corresponding fringe displacement on the sample. This is in practice achieved by inserting an auxiliary linearly birefringent plate (e.g. any commonly available plate $\lambda / 4, \lambda / 10$, etc.) between the polarizer (linear or elliptic) and the sample in such a way that the fast axis of the auxiliary plate is parallel to an axis of the sample which will be temporarily called the $X$ axis. The state $P_{0}$, in general elliptic (Fig.4), is then shifted along the circle $P_{0} E F$ in a counter-clockwise direction about $X$. If $X$ actually is the fast axis of the sample, the direction of motion of $P(z)$ is also counter-clockwise about $X$ and the arc $P_{0} E$ measured in that direction is then smaller. From (2), this means a shift of the fringe system towards the source. In short, one introduces between polarizer and sample any auxiliary retardation plate with a path difference smaller than $\lambda / 2$ and with its known axes approximately parallel to those of the sample. The fringes are shifted towards the source if the fast axis of the sample lies near the fast axis of the auxiliary plate, while they are shifted away from the source if the opposite holds true. This reasoning was found experimentally to be correct, although no illustration of it is given in the experimental part below.

\section{Oblique incidence}

At oblique incidence, one may, in practically every physical situation, consider the two slightly diverging wave normals of the refracted wave fronts as having a common mean direction $\mathrm{O} z$ making an angle $r$ (angle of refraction) with the normal to the entrance facet. The fore- 
going analysis on the Poincaré sphere is still valid, the only difference being that the location of the diameter $M N$ and the value of $\Lambda^{\prime}$ are now functions of $r$. Along the direction of propagation $O z$, the minima of scattered intensity are still separated by $2 \pi / \Delta^{\prime}(r)$. However, the planes of equal phase difference are not normal to $\mathrm{Oz}$ but parallel to the entrance facet. Their trace in a plane normal to the direction of observation constitute the fringe system, so that the fringe width is now

$$
\Lambda(r)=\frac{2 \pi \cos r}{\Delta^{\prime}(r)} .
$$

In the particular case of linear birefringence, the angular dependence of the phase difference per unit distance along the wave normal in a biaxial crystal, say, is of the form

$$
\Delta^{\prime}=\delta^{\prime}=K \sin U_{1} \sin U_{2}
$$

where $K$, in first approximation, is a constant and $U_{1}, U_{2}$ are the angles which $O z$ makes with the respective optic axes. The situation is especially simple in uniaxial crystals $\left(U_{1}=U_{2}=U\right)$, where for a plate cut normally to the optic axis

$$
U=r, \quad \delta_{\perp}^{\prime}=\frac{2 \pi}{\lambda}\left(n_{\varepsilon}-n_{\omega}\right) \sin ^{2} r,
$$

and for a plate containing the optic axis

$$
U=\frac{\pi}{2}-r, \quad \delta_{\|}^{\prime}=\frac{2 \pi}{\lambda}\left(n_{\varepsilon}-n_{\omega}\right) \cos ^{2} r .
$$

Here $n_{\varepsilon}$ and $n_{\omega}$ are respectively the extraordinary and the ordinary refractive indices. In the above crystal sections normal and parallel to the optic axis, the corresponding dependence of the fringe width on the angle of refraction $r$ is therefore, from (3) and (5),

$$
\begin{gathered}
\Lambda_{\perp}(r)=\frac{\lambda}{\left(n_{\varepsilon}-n_{\omega}\right) \sin r \tan r} ; \\
\Lambda_{\|}(r)=\frac{\lambda}{\left(n_{\varepsilon}-n_{\omega}\right) \cos r} .
\end{gathered}
$$

The measurement of $\Lambda_{\perp}(r)$ over a reasonable range provides a fairly precise determination of the magnitude of linear birefringence $\left(n_{\varepsilon}-n_{\omega}\right)$ in a crystal section roughly normal to the optic axis, and this even in presence of circular birefringence, since usually $\delta^{\prime} \gg 2 \varrho^{\prime}$ for sufficiently large values of $r$. A test of that method with the linear birefringence of natural quartz is given below. 
On the other hand, a single measurement of fringe width $\left(\Lambda_{\|}\right)$in a crystal section which contains the optic axis of a uniaxial crystal or the optic plane of a biaxial crystal gives precise values of $\left(n_{\varepsilon}-n_{\omega}\right)$ or $\left(n_{\gamma}-n_{\alpha}\right)$ respectively, where $\gamma$ and $\alpha$ refer to the symmetry axes of the refractive index ellipsoid (indicatrix). As such a section shows the maximal birefringence of a substance, the observed values of $\Lambda_{\|}$may become very small (of the order of a few micron), but that inconvenient is compensated by the low sensitivity of $\Lambda_{\|}$to $r$ at nearly normal incidence and by the correspondingly large tolerance allowed in orienting the sample.

\section{Diffraction by "coarse" centers $(a \approx \lambda)$}

It may be noted that the $90^{\circ}$-Rayleigh scattering is not the only kind which can lead to the observation of birefringence. As a matter of fact, the scattering of a linear vibration at arbitrary values of $\theta$ by centers comparable in size with the wavelength used is also linearly polarized with the plane of vibration parallel or normal to the plane of observation as long as the transmitted vibration near the site of the scattering center lies itself in or normally to that plane (Fig. 5). If the transmitted vibration is at an azimuth $\psi \neq(0, \pi / 2)$ with respect to the plane of observation, the scattered vibration is in general elliptically polarized, although the representing ellipse is in most cases so elongated as to allow a significant extinction by a linear analyzer. In general, the azimuth of the scattered vibration is not the same as that of the transmitted one, since the intensities of the scattered components parallel and normal to the plane of observation are strongly dependent on the ratio $a / \lambda$, on the dielectric properties of the scattering center and on its shape. Even with $\psi=0$ or $\pi / 2$, the most drastic difference with the $90^{\circ}$-Rayleigh scattering is in the intensity of the scattered vibration, which in general does not vanish for $\theta=\pi / 2$, no matter what the azimuth $\psi(z)$ of the transmitted vibration is. The analyzer model must therefore be discarded.

Nevertheless, while the scattered intensity is no longer significantly dependent on the state of polarization of the transmitted vibration, there remains a very strong correlation between the polarization properties of the transmitted vibration and those of the scattered one. One can retain the previous representation of the scattered light by $S$ and $S_{\mathrm{a}}$ on the Poincaré sphere, with the obvious generalization that in the case of forward or backward scattering, $S$ does not directly represent the direction of observation but merely the azimuth of the plane 


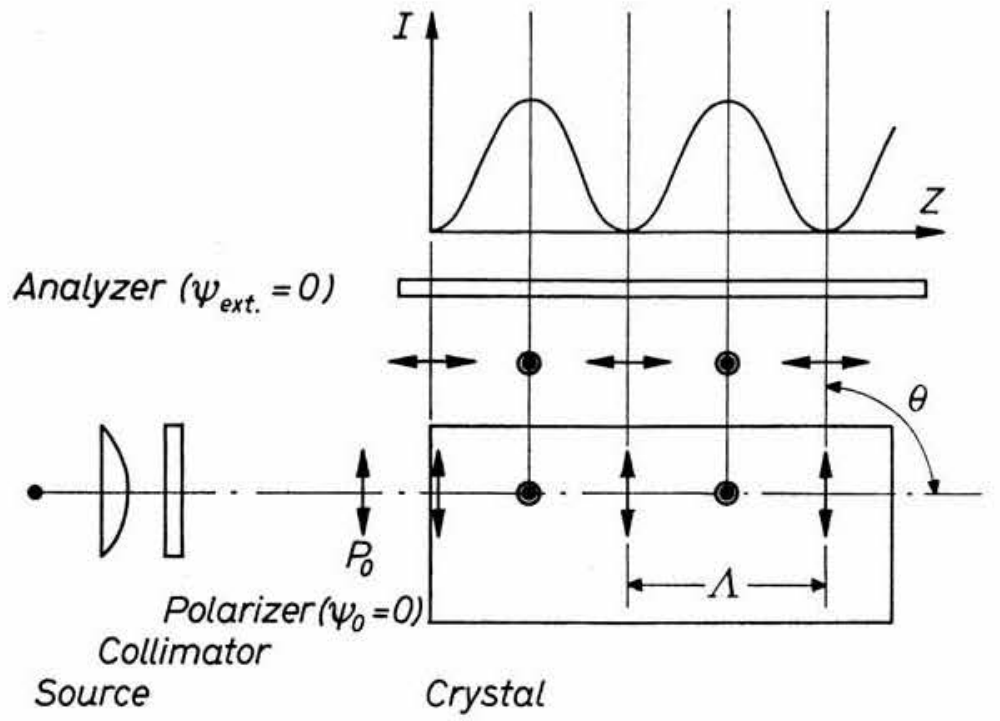

Fig.5. Apparative set up for observing birefringence in the light-scattering configuration. The special case of a linear incident vibration lying in the plane of the paper and scattered by centers with $a \approx \lambda$ is illustrated

of observation. Instead of being the intensity, it is now the state of polarization of the scattered light which is a strong periodic function of $z$, as shown in Fig. 4. Assuming for instance an incident vibration $P_{0}$ linearly polarized in the state $S$ or $S_{\mathrm{a}}$ (Fig. 3), the state $P(z)$ describing the transmitted beam is in general elliptic but it becomes periodically linear at $S$ and $S_{\mathrm{a}}$ and so does the scattered beam also (Fig. 5). It is therefore only necessary to place a linear analyzer in the scattered beam in order to obtain, for the extinction direction in the plane of observation, the same fringe system as in $90^{\circ}$-Rayleigh scattering or, if the analyzer is turned by $90^{\circ}$, a complementary fringe system where the positions of the dark and of the bright fringes are interchanged. In this manner, the same kind of simultaneous analyzation of the directly transmitted beam as the one provided by the scattering centers in the Rayleigh limit is again made possible, and moreover, one is dealing with a much brighter fringe system, due to the $a^{6}$ dependence mentioned earlier for the scattered intensity.

E. Extreme situations; limits of resolution

Due to the finite numerical aperture of the optical system (e.g. microscope objective, camera objective, etc.) used for observing the scattering pattern, on the one hand, or to the large angular field of view which may be subtended by the sample, on the other hand, the finite depth $d$ of the scattering region in the direction of observation 
may have prohibitive effects on the visibility of the fringes if $d>\Lambda$. For photographic purposes, an appropriate field lens may be placed over the sample in order to realize a telecentric system and hence compensate for the otherwise too strong perspective. As it will be shown below however, the perspective effect can be eliminated by making use of surface scattering. It must nevertheless be considered that, even in the case of surface scattering, a high-aperture optical system covers a wide range of values of $\theta$ and of $\psi_{0}$. Since the fringe system is in general shifted and more or less blurred by varying $\psi_{0}$ [see Fig. 3 and equation (2)], and since moreover in the case of Rayleigh scattering the dark fringes are no longer completely extinguished for $\theta=\pi / 2$, the numerical aperture, especially of microscope objectives, must remain limited and a corresponding limit of resolution for the observation of extremely dense fringe systems must be accepted.

From another point of view, it is clear that the grain size of the scattering-center distribution may become a limiting factor when the birefringence is very strong (e.g. $\Lambda_{\|} \approx 2$ micron in calcite). Such a dense fringe system should only be observable in Rayleigh scattering and with a sufficiently high concentration of scattering centers.

The statements just made about the numerical aperture of microscope objectives have their practical counterpart in that, experimentally, the visibility of the fringes for numerical apertures $>0.3$ becomes rapidly too low. This results in the fact that the fringes can in practice be neatly resolved, say, for $\Lambda \gtrsim 2$ micron.

This xtreme situation, how unpractical soever it may be, illustrates by the same token the superiority, in such cases, of the method described earli $r$, which is based on the angular dependence of the birefringence in the vicinity of an optic axis. As a whole, it can be stated that, if the relevant precautions are taken, the light-scattering configuration is capable of dealing with most of the physical cases of general birefringence encountered, from the optical activity of sugar solutions up to the strong linear birefringence of calcite, i.e. in the range

$$
10^{-6}<n_{N}-n_{M}<0.2 \text {. }
$$

\section{F. Interference colours}

The simultaneous analyzation realized by the light-scattering configuration is analogous to the one obtained by placing a wedge of anisotropic material in diagonal position between crossed polarizer and analyzer. In monochromatic illumination, a wedge with angle $\alpha$ pro- 
duces a fringe system, the fringe width $\Lambda_{\mathrm{w}}$ of which is related to the one observed in the light-scattering configuration at normal incidence by

$$
\Lambda_{\mathrm{w}}=\Lambda \cot \alpha
$$

assuming samples of the same substance in the same crystallographic orientation with respect to the direction of propagation of the transmitted beam. The expression (1) for the fringe width can be put in the general form

$$
n_{N}-n_{M}=\frac{\lambda}{\Lambda},
$$

where $\lambda$ is the vacuum wavelength of the radiation and

$$
n_{N}-n_{M}=\left[\left(n_{Y}-n_{X}\right)^{2}+\left(n_{R}-n_{L}\right)^{2}\right]^{1 / 2}
$$

is the birefringence as defined in the introduction. (7) shows that when there is no dispersion of birefringence, the fringe width is proportional to the wavelength. By using a white light source and a colourless sample, the selective extinction of the scattered spectrum is dependent on $z$ and gives rise to the well-known sequence of vivid interference colours graphically represented by the Michel-Lévy chart ${ }^{2,14}$. In practice, of course, the nuances of colour are somewhat affected by the generally encountered dispersion of birefringence and moreover, in the light-scattering configuration, by the spectral dependence of the scattered intensity. The latter, especially in the case of Rayleigh scattering, produces a predominance of the greenish and bluish tones.

\section{Experiments}

\section{A. Apparatus}

The essential pieces of apparatus are shown in Fig. 5. For visual observations at fixed wavelength, a spectral lamp or an incandescent lamp with monochromator are suitable when using surface scattering with $a \approx \lambda$. In the Rayleigh limit however, the available scattering intensity is usually so low as to require the use of a laser (e.g. of the $2 \mathrm{~mW}$ He-Ne type).

As it can be visualized in Fig. 3, the polarizer may be any device yielding linear, elliptic or circular vibrations, although a simple polaroid does away with most situations. When an analyzer is required, a polaroid is again advantageous in photographic recording with large

14 A. Michel-Lívy et A. Lacroix, Les minéraux des roches. Paris 1888. 
angular field of view while a nicol may be preferred in visual observations at low intensity levels.

Quantitative measurements were made both visually and photographically. In the case of strong birefringence, the narrow fringe width was directly measured with a microscope used in a comparator configuration or in connection with calibrated ocular-micrometer scales. Where the birefringence was low enough, the sample was directly photographed together with a calibrated scale and the fringe width was measured on enlargements.

To demonstrate or to quantitatively take account of the variation of birefringence with the direction of propagation, a suitable means of orienting the sample should be provided. According to the size of the specimen, a small goniometer or the rotating stages of a polarizing microscope or of a precision spectrometer all proved to be reliable within their inherent limits of accuracy.

\section{B. Sample preparation}

Hitherto, it has been spoken of a plate throughout the analysis, although this was merely for the sake of remaining inside a well-known context. It is clear that such a geometry is not required here since one is not interested in what happens to the transmitted beam after leaving the scattering region, except perhaps for the fact that it should not produce untolerable amounts of stray light. Essentially, the sample must have two facets: one entrance facet against the incident beam and one observation facet at roughly $90^{\circ}$ to the first one. A high-quality polish for the entrance facet can usually be dispensed with, even when working with a coherent light source, since one does not use the directly transmitted light and since the homogeneity of the scattering pattern is primarily determined by the homogeneity of the scattering-center distribution. As a matter of fact, a finely ground entrance facet wetted with paraffin oil and covered with a glass platelet has given satisfactory results in crystalline quartz. On the other hand, painting the unused faces in black is most effective in eliminating the stray light in the background of the observation facet.

In so far as there is enough bulk scattering in the sample, no further preparation is required. However, with the very perfect quartz crystal described below, it was more convenient to use the scattering at a surface with specified roughness. For that purpose, the observation facet was ground at a small angle to the intended direction of propagation for the transmitted beam, so that the entrance facet and the 
observation facet made a slightly acute angle. The transmitted beam meets then the observation facet at grazing incidence and it must only be so deep along the direction of observation as to illuminate the facet on its whole length. Through fine grinding with alumina from 600 to 3200 mesh or through subsequent partial polishing, the roughness could easily be adjusted to the conditions $a \approx \lambda$ or $a \ll \lambda$.

The biaxial crystal of monoclinic $\mathrm{Eu}_{2} \mathrm{SiO}_{4}$ used in this work has been shaped to a platelet $(2 \times 1.5 \times 0.5 \mathrm{~mm})$, where the smallest dimension is along one of the optic axes for red light $(\lambda=632.8 \mathrm{~nm})$ and where one of the faces is parallel to the optic plane. All the faces have been polished with 1/4-micron diamond paste on a silk cloth impregnated with thermoplastic (the latter prevents an excessive rounding off of the edges when such small surfaces are worked on). This allowed surface scattering of the Rayleigh type to be observed. The measurements for directions of propagation in the vicinity of one optic axis could however be made by means of bulk scattering of the Rayleigh type.

The data reported below for EuTe have been gained from bulk scattering. The crystal, which is polished on all sides, has been embedded in plastic and surrounded by glass platelets, though this precaution was only dictated by the chemical instability of the surface in a humid atmosphere. A metallic screen with a $1 \times 1 \mathrm{~mm}$ opening has been attached to the entrance facet in order to limit the transmitted beam more sharply and to minimize the stray-light effects accordingly.

\section{Results}

In order to illustrate some highlights of the light-scattering configuration for observing and measuring birefringence, the following cases with various scattering conditions have been selected:

a) the circular (optical activity and rotatory dispersion), the elliptic and the linear birefringence of quartz;

b) the linear birefringence of europium(II)-orthosilicate;

c) the magnetic circular birefringence (Faraday rotation) of europium telluride at room temperature.

\section{General birefringence of quartz}

In spite of the fact that quartz ranges among the substances with the highest values of optical activity, it can be said to have a medium birefringence in the general scheme considered here, since the circular 


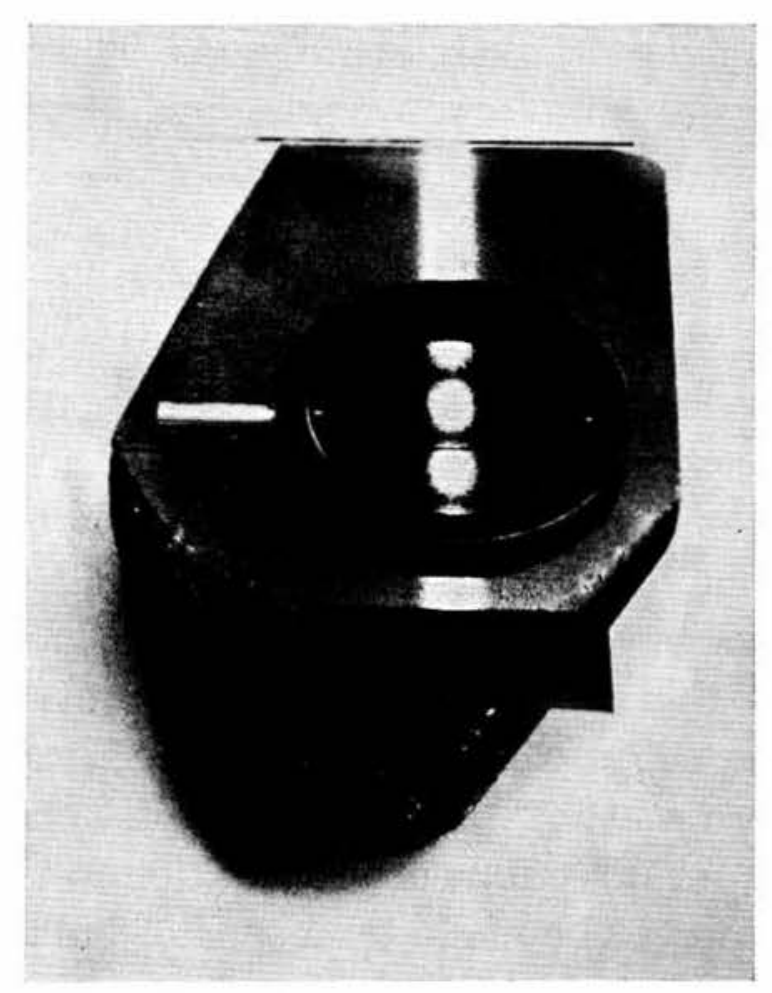

Fig. 6. Optical activity of quartz along the optic axis in forward surface scattering $\left(\theta=45^{\circ}\right)$ with $a \approx \lambda$

birefringence $\left(\left|n_{R}-n_{L}\right| \approx 0.00007\right)$ is nonetheless two orders of magnitude smaller than the linear birefringence $\left(n_{\varepsilon}-n_{\omega}=0.009\right)$. The corresponding observable fringe widths lie in the range $0.1-10 \mathrm{~mm}$ and can be usually observed with the unaided eye or directly photographed with close-up lenses. That substance, which is available in very perfect form, lends itself therefore readily to a thorough testing of the lightscattering configuration for measuring birefringence. The specimen investigated hereafter, obtained from Ebauches SA, Neuchâtel, Switzerland, is a brasilian left-handed quartz of the grade used for high-Q oscillators in the watch industry.

\section{a) Optical activity}

Figure 6 is a photograph of the sample under ordinary ambiant illumination with a laser beam along the optic axis and with forward scattering $\left(\theta=45^{\circ}\right)$ of the type $a \approx \lambda$. The incident beam is linearly vibrating in the plane of observation $\left(\psi_{0}=0\right)$ and the analyzer lying on the observation facet has its extinction direction also in the plane of observation $\left(\psi_{\text {ext }}=0\right)$. As mentioned in section IID, one notices that the analyzer has the same effect as though it were placed along the 


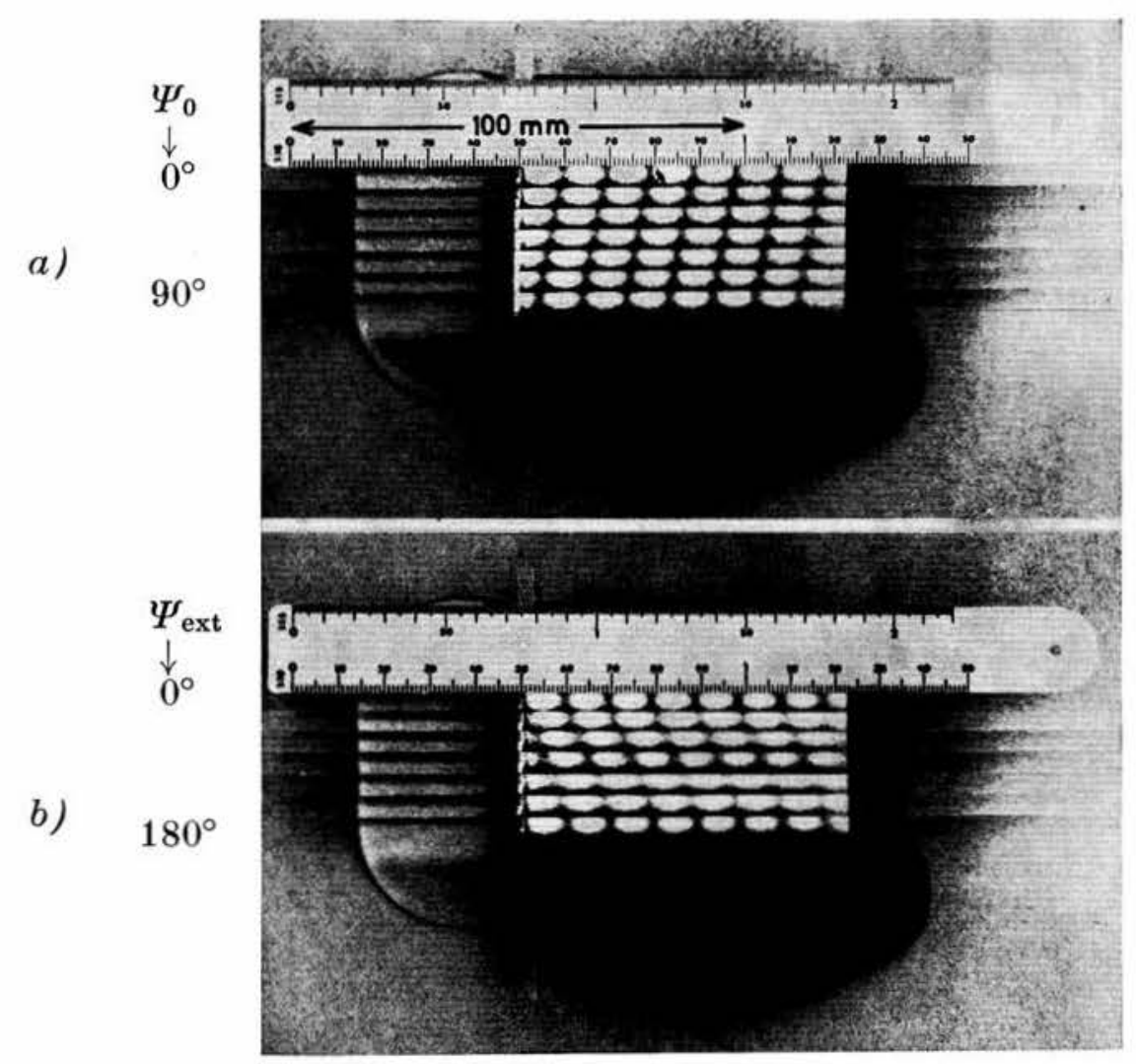

Fig.7. Composite pictures showing the optical activity of a left-handed quartz along the optic axis in $90^{\circ}$ surface scattering with $a \approx \lambda: a$ ) shift of the fringe system towards the source, on the left, by rotating the linear polarizer in a positive direction from zero to $90^{\circ}$ in steps of $15^{\circ}$ while holding the analyzer fixed; b) opposite shift produced by rotating the analyzer in a positive direction from zero to $180^{\circ}$ in steps of $30^{\circ}$ while holding the polarizer fixed. Both shifts are reversed in a right-handed sample

transmitted beam at any site under consideration and with the same orientation of its extinction direction with respect to the plane of observation $\left(\psi_{\text {ext }}=0\right)$. It would then extinguish the transmitted beam at every place $z$ where $\psi(z)=0$. This is verified in Fig. 7 a, which is a composite picture of $90^{\circ}$ scattering made under similar conditions, except for the azimuth $\psi_{0}$ of the incident linear vibration $P_{0}$, which was varied from zero to $+90^{\circ}$ in steps of $15^{\circ}$ (the azimuth is counted positive in the counter-clockwise sense for an observer looking against the oncoming beam). The corresponding picture for a constant incident vibration with $\psi_{0}=0$ and for the analyzer being rotated in steps of $30^{\circ}$ from zero to $180^{\circ}$ is shown in Fig. $7 \mathrm{~b}$. It appears in this case that the simultaneous-analyzation analogue is strictly valid only for the limiting conditions $\psi_{\text {ext }}=0$ and $\psi_{\text {ext }}=90^{\circ}$, while the scattered beam for $\psi(z)=0$ and $90^{\circ}$.is no longer completely linearly polarized. 
For the purpose of a quantitative check, the pictured scale has been calibrated against a precision cathetometer and the 50-mm mark was ajusted exactly over the edge of the entrance facet in order to provide an estimate of the perspective error due to oblique rays in taking the picture. The $2^{\circ}$ angle between the optic axis and the ruler, which lies flat on the sloping observation facet, has been taken into account. By calculating the angles of refraction paired for equal values of $\Lambda$, the symmetry axis of the elliptic birefringence (see below) and hence the

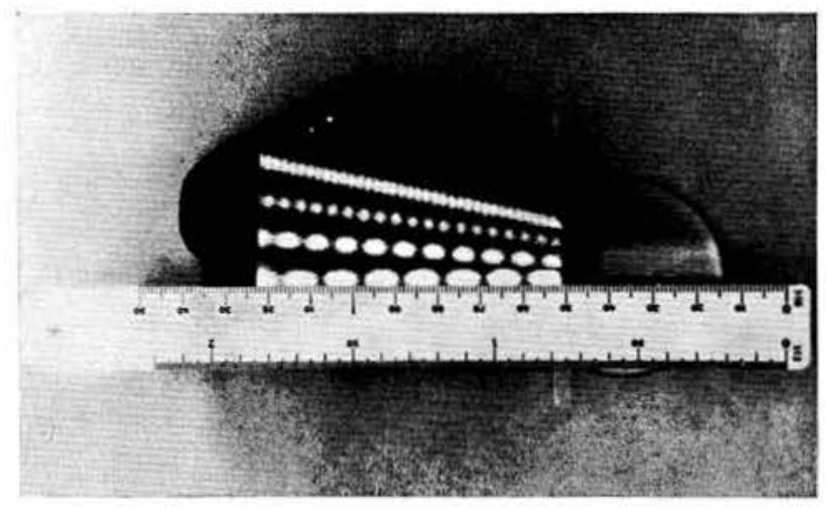

Fig. 8. Angular variation of the elliptic birefringence of quartz in the vicinity of the optic axis (composite picture)

optic axis was found to make an angle of $10^{\prime}$ with the normal to the entrance facet. In units of rotatory power, the residual systematic errors added up to less than $0.01 \% \mathrm{~mm}$ for the five fringes in the center of the photograph (Fig. $7 \mathrm{a}$ ) between the mm marks 65 and 120. Fourty different fringe widths were individually measured in Fig. 7 a with a standard deviation from the mean equal to $0.04 \% \mathrm{~mm}$. The rotatory power amounts then to

$$
\left(\varrho^{\prime}\right)_{27^{\circ} \mathrm{C}}^{632.8 \mathrm{~nm}}=(18.76 \pm 0.05)^{\circ} / \mathrm{mm},
$$

while the corresponding interpolated value from the data of the International Critical Tables reads $18.72 \% \mathrm{~mm}$.

Figure 7 a gives also information about the hand of the crystal. It is found indeed that a rotation of the polarizer in the positive direction produces a shift of the fringes towards the source (situated on the lefthand side of the picture), so that according to the explanation of paragraph IIC 2 the sample is left-handed. The opposite shift was actually observed in the sample of Fig. 10, which is right-handed.

It can be seen moreover from Fig. $7 \mathrm{~b}$ that the simultaneous analyzation provided by the light-scattering configuration with $a \approx \lambda$ 
(hence with the use of a separate analyzer) also allows the sign of the azimuthal rotation of the transmitted vibration to be determined in a way analogous to the traditional one, namely by turning the analyzer so as to restore the position of the fringe system when it has been shifted by a small positive rotation of the polarizer. In the example shown, a positive (counter-clockwise) rotation of the analyzer is then required, so that in accordance with the usual conventions the sample is left-handed.

\section{b) Elliptic and linear birefringence}

Figure 8 illustrates the angular dependence of the elliptic birefringence of quartz in the vicinity of the optic axis. The incident beam is no longer in the plane of observation but still vibrating parallel to it, so that $\psi_{0}=0$ immediately behind the entrance facet in the sample. The linear analyzer in front of the camera was at the azimuth $\psi_{\text {ext }}=0$. For a quantitative test of Eq. (6a), a line was drawn across the observation facet parallel to the edge of the entrance facet and the angles of incidence for which a dark fringe was centered on that line were read on the vernier of the spectrometer used as sample holder. Those angles were found to be easily reproducible within $\pm 1^{\prime}$. In order to eliminate the errors arising from the misorientation of the optic axis and from the lack of parallelism between the reference line and the entrance facet, the measurements were made on either side of the optic axis, the angles of refraction were individually computed from the known value of ordinary refractive index $n_{\omega}$ and the mean value $r$ of each pair of angles of refraction corresponding to a given $\Lambda$ was taken. A plot of the reciprocal fringe width as a function of $\sin r \tan r$ is shown in Fig. 9. If there were only linear birefringence, one would have according to $(6 \mathrm{a})$ a straight line passing through the origin with the slope $\left(n_{\varepsilon}-n_{\omega}\right) / \lambda$. The presence of circular birefringence is shown by the departure from the limiting straight line. Away from the optic axis, the circular part of the elliptic birefringence is seen to become rapidly undetectable as soon as the linear part gets a few times stronger, as it is to be expected from the quadratic addition law in (1). The experimental straight line obtained for sufficiently high values of $r$ provides therefore a simple way of measuring linear birefringence even in presence of a weaker circular contribution. In this case, one finds

$$
\left(n_{\varepsilon}-n_{\omega}\right)_{27^{\circ} \mathrm{C}}^{632.8 \mathrm{~nm}}=0.00899 \pm 0.00005
$$




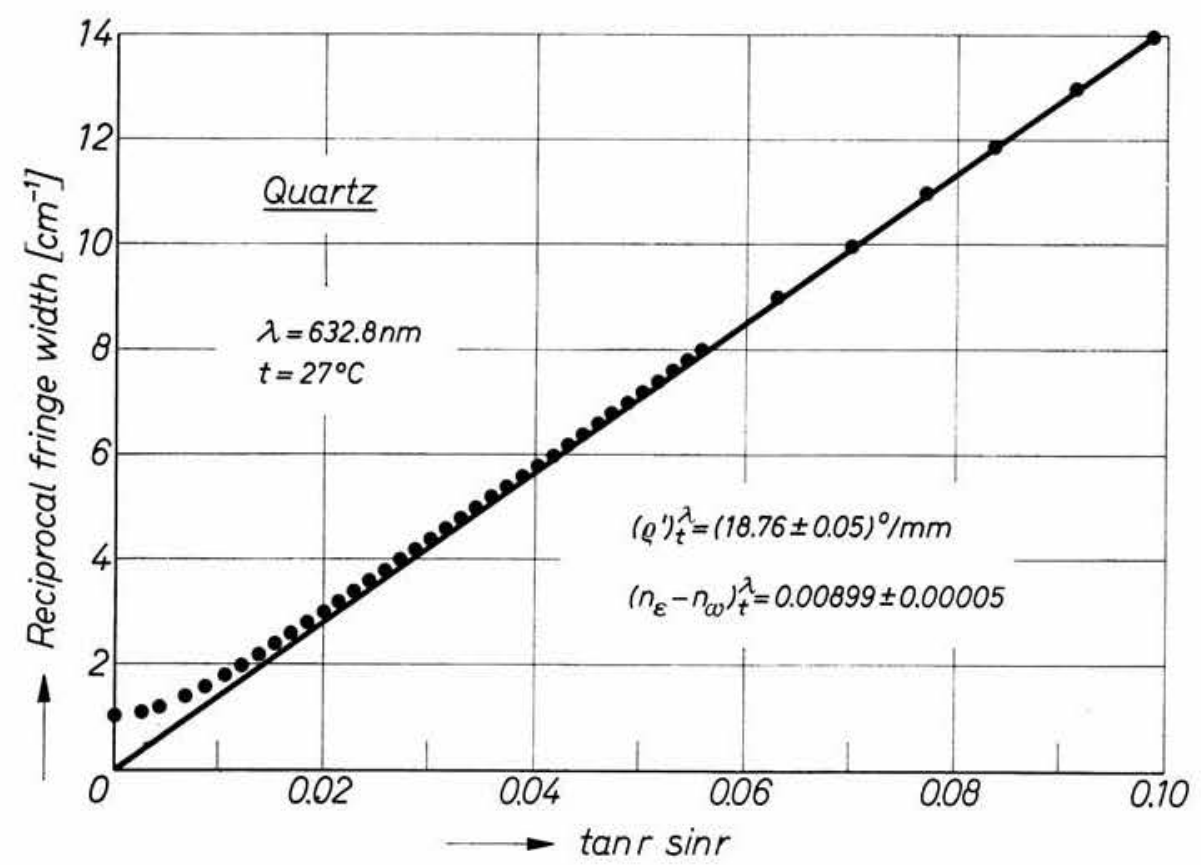

Fig.9. Quantitative data corresponding to Fig. 8 and Eq. (6a). • : experimenta] points; — : theory when only linear birefringence is present

The literature value ${ }^{15}$ interpolated for that wavelength with an appropriate temperature correction amounts to 0.00904 .

The same kind of measurement in a less perfect specimen of righthanded smoke quartz gave $\left(n_{\varepsilon}-n_{\omega}\right)=0.00902$. Figure 10 shows that that sample actually consists of several individuals grown with verylow-angle grain boundaries. The sensitivity and the local-analyzation character of the light-scattering configuration are well illustrated by that application example. From the actual fluctuations in fringe width and from the angular variation of birefringence, the optic axes of the various individuals are found to lie within $\pm 20^{\prime}$ from one another.

\section{c) Rotatory dispersion}

Although the dispersion of linear birefringence usually refers to the spectral dependence of the difference in the refractive indices $n_{X}$ and $n_{Y}$ of a linearly birefringent plate $X Y$, the rotatory dispersion refers directly to the spectral dependence of the rotatory power $\varrho^{\prime}(\lambda)$ and hence to the specific phase difference

$$
2 \varrho^{\prime}(\lambda)=\frac{2 \pi}{\lambda}\left[n_{R}(\lambda)-n_{L}(\lambda)\right]=\frac{2 \pi}{\Lambda} .
$$

$15 \mathrm{~J}$. W. GIFFord, The refractive indices of fluorite, quartz and calcite. Proc. Roy. Soc. [London] 70 (1902) 329-340. 
A new method to measure general birefringence in crystals

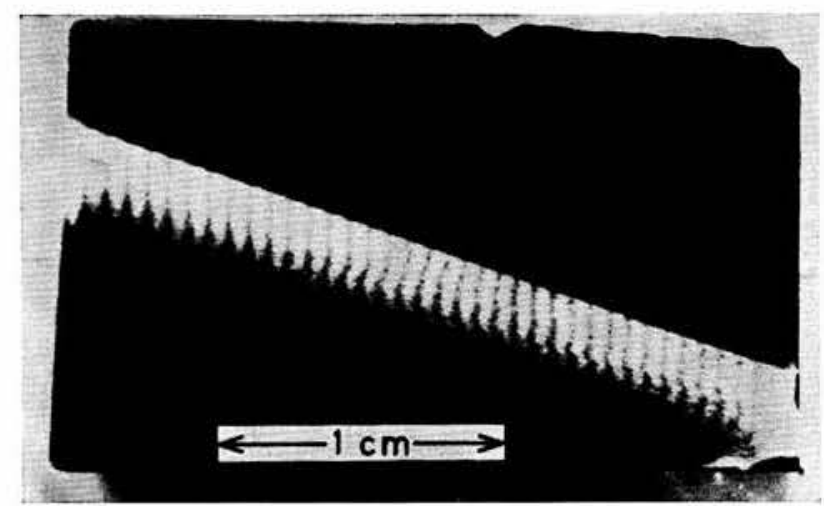

Fig.10. Elliptic birefringence in a quartz sample consisting of several individuals with nearly the same crystallographic orientation

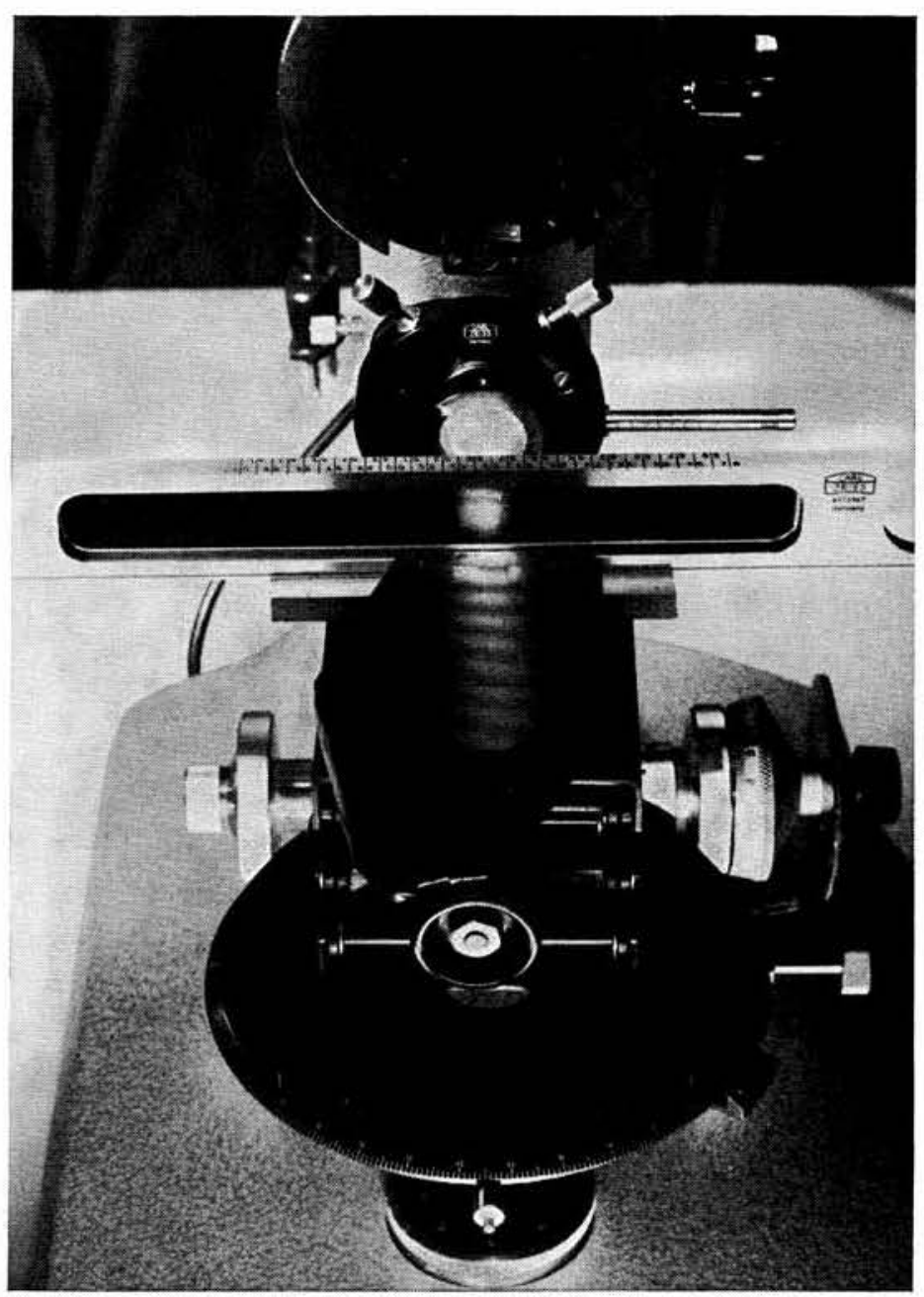

Fig.11. Rotatory dispersion of quartz in surface scattering $\left(\theta=45^{\circ}, a \approx \lambda\right)$ with a $60 \mathrm{~W}$ microscope bulb

In contrast with the cases of linear, elliptic and circular birefringence as they were defined in the introduction, the rotatory dispersion $\varrho^{\prime}(\lambda)$ includes implicitely the dispersion of circular birefringence and the factor $\lambda$ of Eq. (7). It is mainly that factor $\lambda$ instead of being the disper- 
sion of circular birefringence proper which is responsible for the display of interference colours connected with rotatory dispersion.

Figure 11 shows in black and white by means of surface scattering the spectral dependence of the rotatory power or, along any line parallel to the entrance facet, the spectral decomposition of the interference colours at that place. The collimated beam $(2.5 \mathrm{~cm}$ in diameter $)$ from a 60 watt microscope lamp with tungsten filament is aimed under normal incidence at the entrance facet of the sample already shown in Figs. 6 to 8. A graded interference filter with constant gradient of wavelength is placed near the entrance facet in such a way as to transmit the spectral range $570-630 \mathrm{~nm}$ over the width of the beam. Since the dispersion of circular birefringence of quartz is weak in that spectral range as compared to the change in $\lambda$, the leading term for $\Lambda$ in (9) is in the first power of $\lambda$, so that for the small range considered here the scattering pattern actually seen in Fig.11 is an array of straight fringes diverging from some point in the plane $z=0$ of the entrance facet.

The interference colours arising from rotatory dispersion have long been the object of a classical demonstration experiment in this Institute of Technology, where the collimated beam from a carbon are is sent through a saturated sucrose solution containing some scattering additive in suspension. It does not seem to have been realized however that the phenomenon is also observable in surface scattering along the optic axis of a finely ground piece of quartz with no more instrumentation than two polaroids and a pocket flashlight. It is clear that the optic axis is not the only direction exhibiting interference colours: in fact, any direction of propagation does, in accordance with (7). From (8) though, and from the rapid increase of the linear birefringence for directions away from the optic axis, the elliptic birefringence of quartz $\left(n_{N}-n_{M}\right)$ in these directions is soon larger than the circular one and a corresponding scaling down of the interference pattern takes place. Accordingly, by placing the flashlight bulb very close to the entrance facet, the rays diverging from it make the isochromatic contours appear bell-shaped about a facet normal passing through the filament.

\section{Linear birefringence of europium(II)-orthosilicate}

The room-temperature phase of pure $\mathrm{Eu}_{2} \mathrm{SiO}_{4}$ is monoclinic and usually shows polysynthetic twinning on the (100) and (001) planes ${ }^{16}$.

16 R. Verreault, Crystallographic, optical and magnetic properties of $\mathrm{Eu}_{2} \mathrm{SiO}_{4}$. Physik kondens. Materie 14 (1971) 37-54. 


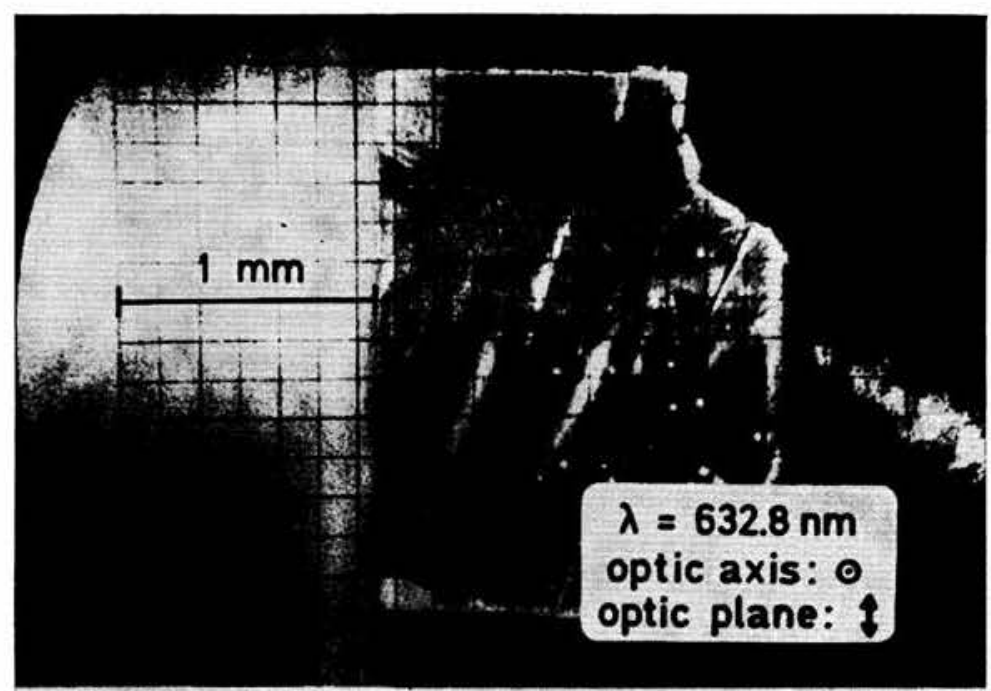

a)

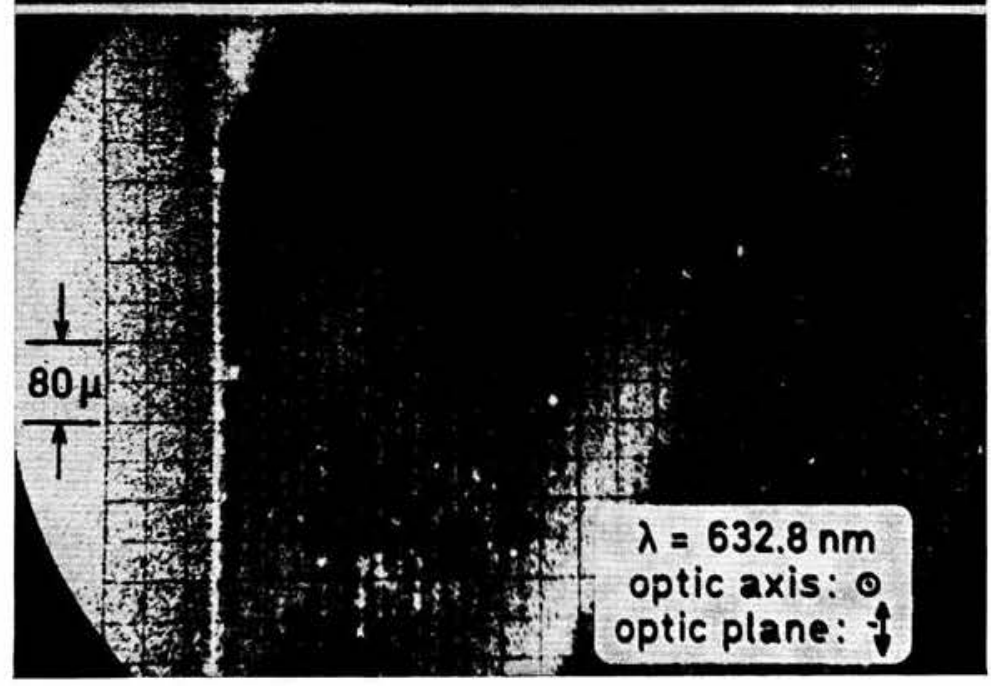

b)

Fig.12. Linear birefringence of monoclinic $\mathrm{Eu}_{2} \mathrm{SiO}_{4}$ in $90^{\circ}$ Rayleigh scattering at the optically-polished observation facet: a) overall view including the trace of the laser beam; b) detail of the scattering surface

The crystals are biaxial positive and optically inactive. Figure $12 \mathrm{a}$ is a photomicrograph giving a general view of a single crystal which has been mechanically detwinned.

\section{a) Propagation normal to the optic plane}

Referring to the description of section IIIB, the observation facet in Fig. 12 lies normal to an optic axis for red light and the entrance facet, on the left, is the face parallel to the optic plane. The light beam from a $2 \mathrm{~mW} \mathrm{He}-\mathrm{Ne}$ laser enters the sample at normal incidence. It is linearly polarized with its plane of vibration in the plane of observation. In that configuration the maximal linear birefringence $\left(n_{\gamma}-n_{\alpha}\right)$ is measured. Apart from a few dots and stripes due to parasitic scatter- 
ing from inclusions and from remaining dislocations at the sites of former twin planes, the main part of the scattered light is of the Rayleigh type at the optically polished observation facet. In order to minimize the amount of stray light and of bulk scattering, the laser beam has been shaped to a shallow sheet just below the observation facet by making use of appropriate screens. Figure $12 \mathrm{~b}$ is a close-up view of the same sample showing a part of the scattering surface. Along the whole path of $1.5 \mathrm{~mm}$ covered by the observation facet, some fringes have been counted with a uniform fringe width of 18.80 micron. This corresponds to the birefringence value

$$
\left(n_{\gamma}-n_{\alpha}\right)_{25^{\circ} \mathrm{C}}^{632.8 \mathrm{~nm}}=0.0337 \pm 0.0003 .
$$

\section{b) Propagation approximately in the optic plane}

In Fig. 13, the same crystal has been turned by approximately $90^{\circ}$ so that the former entrance facet is now the observation facet and that the optic plane practically coincides with the plane of the paper. The narrow portion of the apparent scratch below the trace of the beam is the trace of a former twin plane on the observation facet. It runs parallel to the crystallographic $b$ axis, which is at the same time the $\gamma$ axis of the indicatrix while its $\beta$ axis is nearly the direction of observation. The laser beam, which comes from the left, has been weakly focussed to a diameter of 30 micron and aimed at a region of the crystal which is relatively free from severe imperfections. One is therefore dealing with bulk scattering of the Rayleigh type. The incident linear vibration lies in the plane of observation inside the sample just after leaving the entrance facet.

As it can be seen in Fig. 13, the crystal has been slightly tilted to the right about an axis in the plane of the paper. This precaution can be justified by looking back at Fig. 3 in considering $90^{\circ}$-Rayleigh scattering. When the optic plane lies exactly normally to the direction of observation, the latter coincides with the $\beta$ axis of the indicatrix and is therefore a principal direction (state $X$ or $Y$ in Fig. 3) of the linearly birefringent plate for any direction of propagation lying in the optic plane. This means that, on the Poincaré sphere, $S$ always coincides with either $X$ or $Y$ and that the great circular are $P S$ has a constant length independently of $z$. The scattered intensity is therefore uniform along the whole transmitted path, no matter what the values of $P_{0}$ and $P(z)$ are, and consequently no fringe system whatsoever can be observed. This reasoning actually proved to hold true experimentally. 


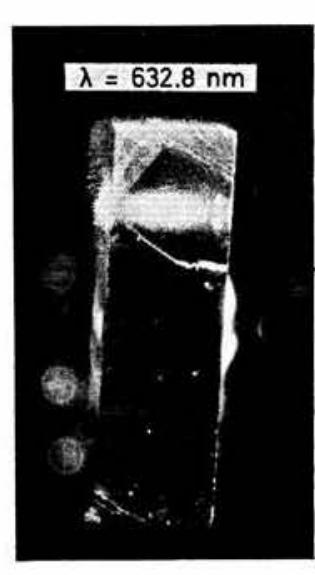

a)

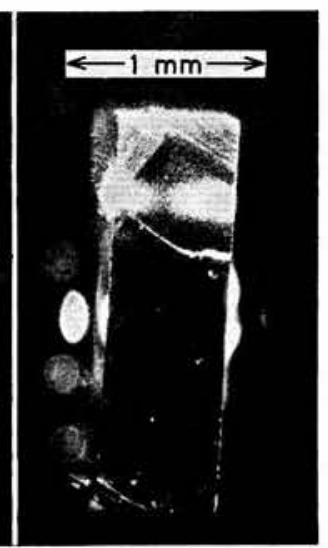

b)

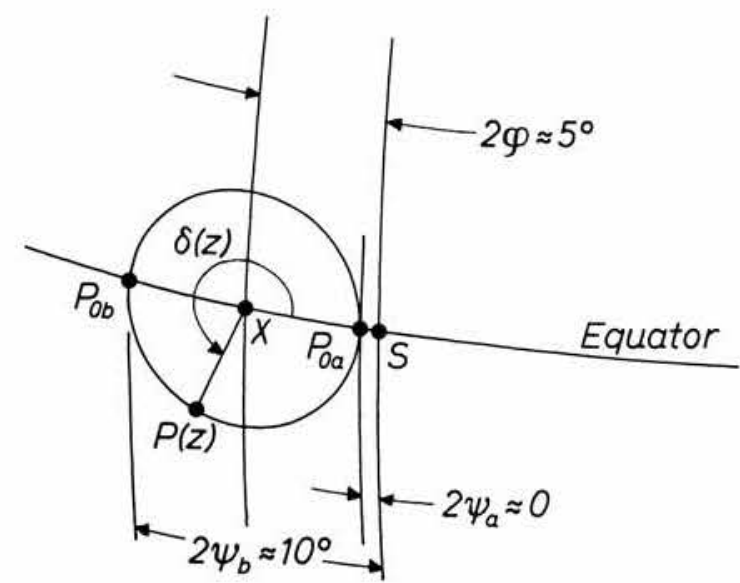

c)

Fig.13. Weak linear birefringence of $\mathrm{Eu}_{2} \mathrm{SiO}_{4}$ near one optic axis. Bulk scattering of the Rayleigh type is used. The respective azimuths $\psi_{\mathrm{a}}$ and $\psi_{b}$ of the incident linear vibrations $P_{0 \mathrm{a}}$ and $P_{0 b}$ just behind the entrance facet for the cases a) and b) are shown in c). This illustrates the conditions for the maximum visibility of a fringe system with non-maximum brightness

A possible way of surrounding the difficulty is to use directions of propagation in a plane slightly tilted with respect to the optic plane, so that the laser beam meets the optic plane, say, from underneath at an angle of a few degrees (Figs. 13a and $13 \mathrm{~b}$ ). For a general direction of propagation in that new plane, the direction of observation $S$ is no longer parallel to the principal directions of vibration $X$ or $Y$; in fact it makes a small angle with one of them. It can be shown in a firstorder approximation that for an optically positive crystal $S$ is near the faster state $X$ on the Poincare sphere when the projection of the direction of propagation on the optic plane lies in the obtuse optic angle (here the one containing the $\alpha$ axis), while it is in the vicinity of the slower state $Y$ when that projection lies in the acute optic angle (containing the $\gamma$ axis). One has thus the situation illustrated in Fig. $13 \mathrm{c}$. The incident linearly polarized vibration is in the state $P_{0 \mathrm{a}}$ in the case of Fig. 13a. Since the optic axis is normal to the entrance facet, the small non-zero angle of incidence makes that there is a small amount of birefringence present for the path followed by the transmitted beam and accordingly the value of $\Lambda$ is just about equal to the crystal thickness (Fig. 13a) instead of being infinite. It was then verified experimentally with Fig. $13 \mathrm{~b}$ that the azimuth of the linearly polarized incident vibration $P_{0 b}$, which yields the complementary fringe system with the first dark fringe at $z=\Lambda / 2$ and with a maximum of fringe visibility, actually is as in Fig. $13 \mathrm{c}$, where $\psi_{b} \approx 5^{\circ}$ instead of the $45^{\circ}$ 


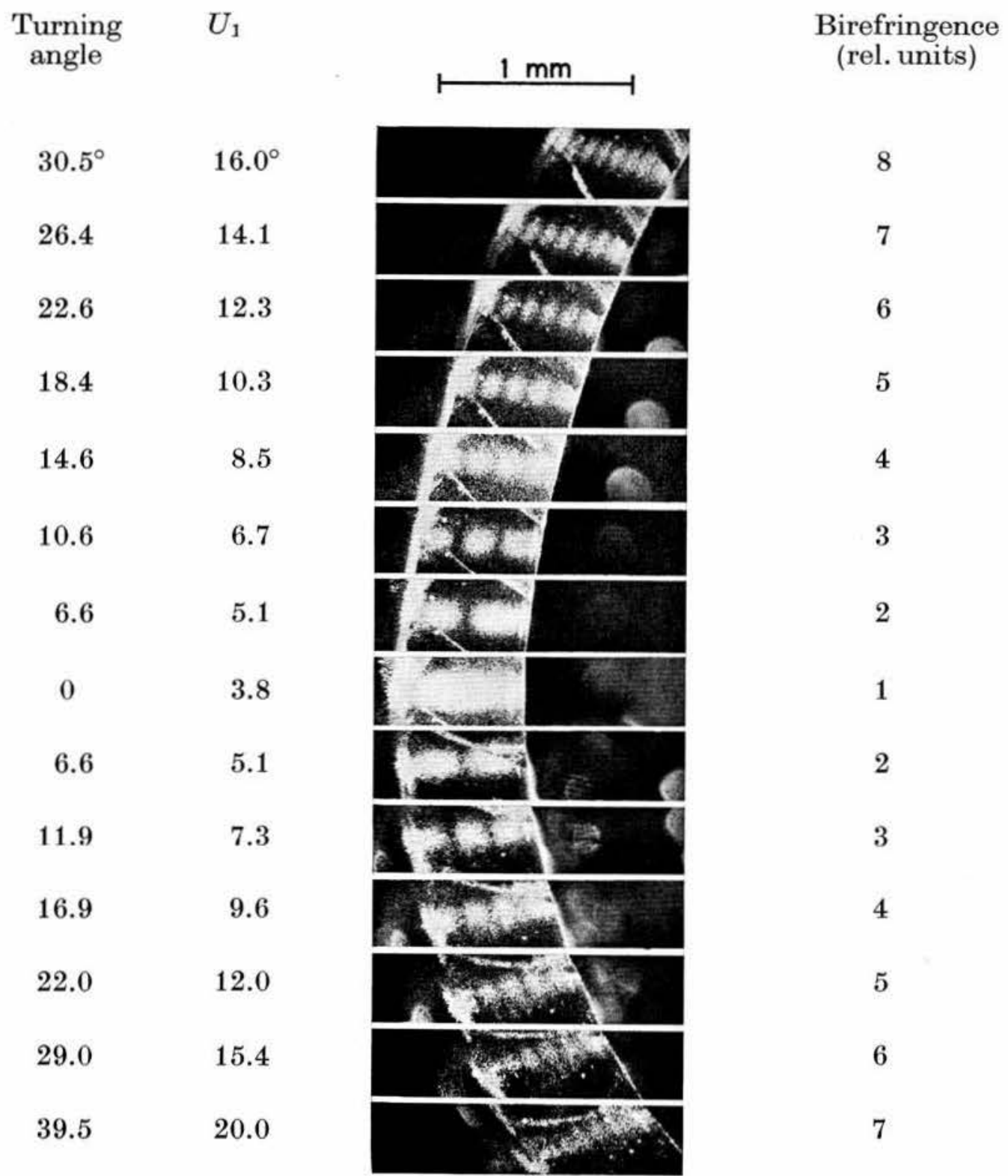

Fig.14. Composite picture showing the asymmetrical angular dependence of linear birefringence about one optic axis in a plane near the optic plane of a biaxial crystal $\left(\mathrm{Eu}_{2} \mathrm{SiO}_{4}\right)$. The incident polarization states correspond to Fig. 13a

azimuth stated by CHANDRASEKHARAN ${ }^{8}$. By choosing azimuths away from those of $P_{0 \mathrm{a}}$ and $P_{0 b}$, the fringe system disappears rapidly.

With the above configuration, it is now possible to study, at least qualitatively, the angular dependence of the linear birefringence of a biaxial crystal in the optic plane on either side of an optic axis. In Eq. (4), let $U_{1}$ be the angle between the refracted beam and that optic axis $\left(O A_{1}\right)$ which is normal to the entrance facet; hence $U_{1}=r$, the angle of refraction. From (3), (4), (7) and (8), one has

$$
\left(n_{Y}-n_{X}\right)=\frac{\lambda}{\Lambda}=\frac{\lambda}{2 \pi} K \tan U_{1} \sin U_{2} .
$$


As $\sin U_{2}$ takes different values for two directions of propagation symmetrically disposed at an angle $U_{1}$ on either side of $O A_{1}$, the angular dependence of $\left(n_{Y}-n_{X}\right)$ about $O A_{1}$ is expected to be asymmetrical, being more rapid for directions of propagation on the side of the obtuse optic angle, where $U_{2}$ is larger in the vicinity of $O A_{1}$. This is verified experimentally, as shown by the composite picture of Fig. 14. This picture is composed of the scattering region of Fig. 13a under various angles of incidence. The first column of numbers gives the turning angle of the crystal in the plane of the paper; that angle is practically equal to the angle of incidence at the higher values. The second column shows the corresponding exact angles of refraction. The third one gives the number of intensity oscillations over the whole path; this is a direct measure of the actual birefringence encountered along the given path. One sees that for a given value of birefringence, a smaller angle of incidence and hence of refraction is necessary (rapid variation) when the refracted beam is located in the obtuse optic angle (upper part of the composite picture), and vice versa.

\section{Magnetic circular birefringence of EuTe}

It might not be at once obvious that while the circular birefringence $\left(n_{R}-n_{L}\right)$ of most opticaliy active materials found in nature is several orders of magnitude lower in value than the linear birefr.ng nce usual y encountered, the spontaneous magnetic circular birefringence of most transparent ferromagnetic semi-conductors discovered in the last decade takes values typically in the range $0.01<\left|n_{R}-n_{L}\right|<0.1$ at the short-wavelength limit of their transparent region (e.g. EuO : 0.06; $\left.\mathrm{CrBr}_{3}: 0.01 ; \mathrm{Eu}_{2} \mathrm{SiO}_{4}: 0.02\right)$. Although these values pertain to temperatures below the Curie point, i.e. well below room temperature, it is nevertheless possible to induce some $10 \%$ of that at room temperature by applying magnetic fields of the order of $30 \mathrm{kOe}$ (Faraday effect).

As an example of a material showing a strong paramagnetic circular birefringence, we have chosen EuTe. Although this substance orders antiferromagnetically at low temperatures with a value of $n_{R}-n_{L}$ $\approx-0.01$ near the Néel point $\left(9.64^{\circ} \mathrm{K}\right)$, its room-temperature Verdet constant is comparable to those of the other europium chalcogenides and it has the advantage of being transparent in the visible. The circular birefringence reaches -0.0007 at room temperature for a wavelength of $632.8 \mathrm{~nm}$ and with an applied field of $30 \mathrm{kOe}$. This is one order of magnitude higher than the natural circular birefringence of quartz. 


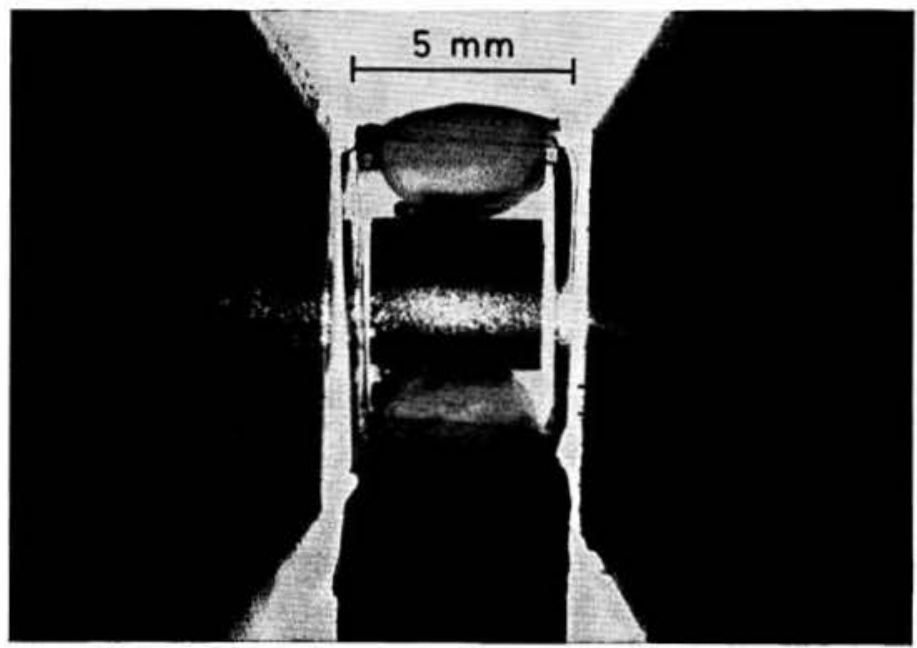

Fig.15. Experimental set up for the Faraday effect of EuTe in Rayleigh bulk scattering. The red-transparent crystal between the perforated pole pieces of an electromagnet appears black against the white background $(H=0)$

Figure 15 shows a single crystal of EuTe between the perforated pole pieces of an electromagnet, though with no applied field. The laser beam, which is linearly vibrating in the plane of the paper, enters the sample from the left and undergoes Rayleigh scattering in the bulk. No analyzer has been used between sample and camera. The nonuniform scattered intensity appears to be only the result of an inhomogeneous concentration of scattering centers.

The effect of gradually applying the magnetic field is shown by the composite picture of Fig.16, which is made up of the central part of Fig. 15 for various fields. From the known azimuth of the incident vibration ( $P_{0}$ coincides with $S_{\mathrm{a}}$ in Fig. 3 ), the first dark fringe, which is at $z=\Lambda / 2$, indicates a $90^{\circ}$ rotation of the plane of vibration within the sample while the subsequent dark fringes correspond to additional $180^{\circ}$ increments of rotation. Using (9), the law of proportionality between the Faraday rotation and the applied field leads to the relation

$$
\frac{\pi}{\Lambda}=V H \text {, }
$$

where $V$ is the Verdet constant. The various values of $\Lambda$ tabulated in Fig. 16 have been directly measured on the individual photographs using the precisely known length of the crystal as a standard. A plot of the reciprocal fringe width as a function of the applied field is shown in Fig. 17. The slope of the best fitting straight line yields the Verdet constant

$$
(V)_{300^{\circ} \mathrm{K}}^{632.8 \mathrm{~nm}}=-(3.79 \pm 0.04) \mathrm{min} / \mathrm{Oe} \mathrm{cm}
$$


$\mathrm{H}$

$\Lambda$

$3.98 \mathrm{kOe} 6.75 \mathrm{~mm}$

7.90

3.37

15.5

1.80

20.6

1.38
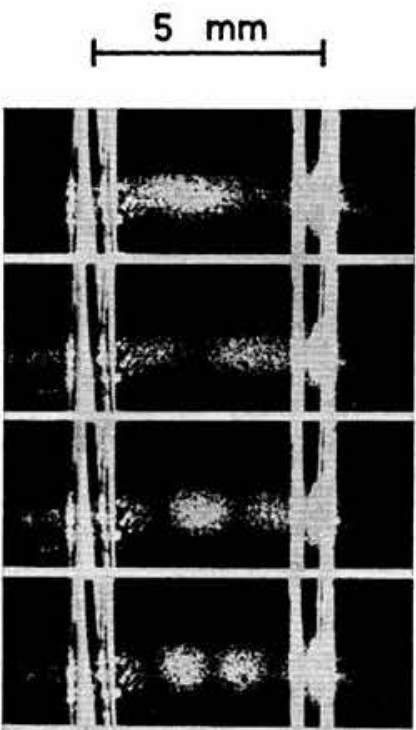

23.3

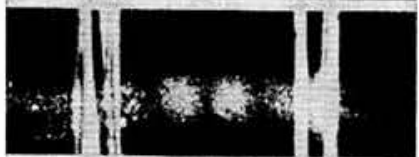

25.2

1.12

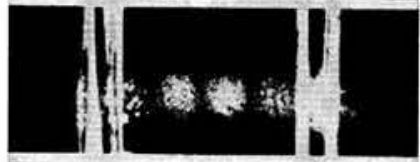

26.4

1.08

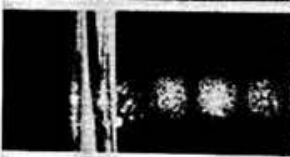

28.5

1.00

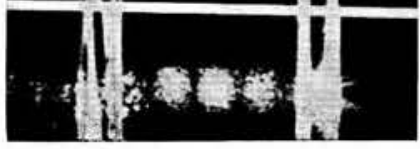

Fig.16. Composite picture showing the variation of the magnetically induced circular birefringence as a function of the applied field

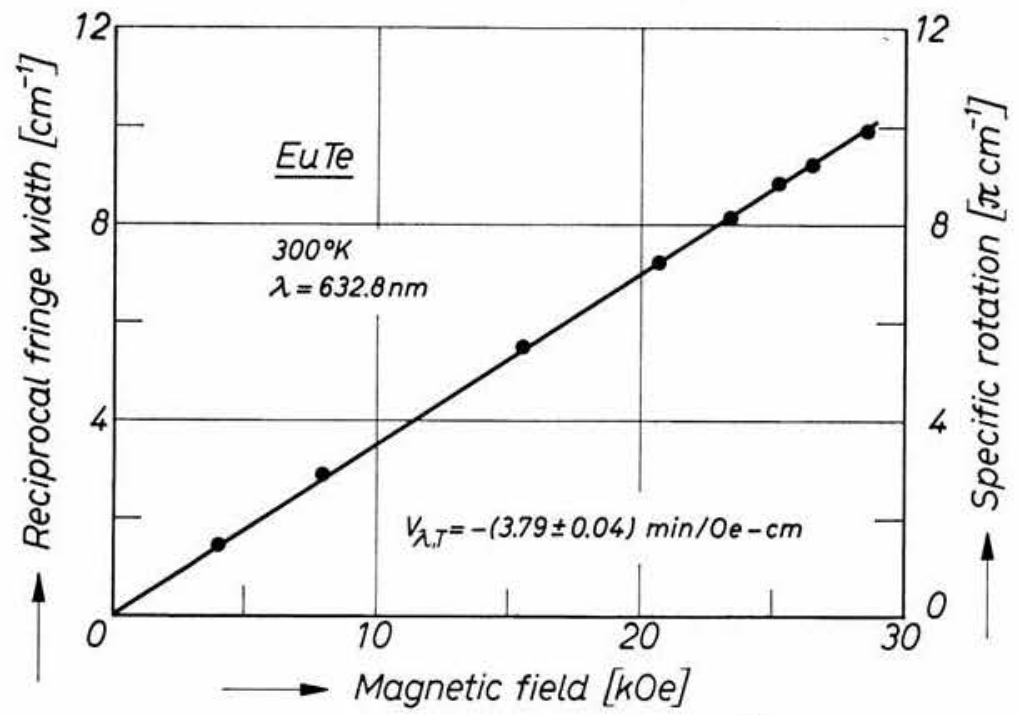

Fig. 1 7. Test of Verdet's law in the light-scattering configuration 
where the minus sign was determined as shown in Fig. 7 a. In an earlier measurement on the same crystal with the conventional method, the value $-(3.79 \pm 0.02) \mathrm{min} / \mathrm{Oe} \mathrm{cm}$ had been obtained.

\section{Conclusion}

The present development of the light-scattering configuration for measuring birefringence now involves very simple experiments in that field of crystal optics. One of the most straight forward applications of the method should be in student-demonstration experiments on the properties of polarized light in crystals. As a matter of fact, the huge Faraday rotation of EuTe at room temperature has been directly picturized on an internal television system in this Institute of Technology. This turned out to be an impressive way of illustrating a lecture on magnetism.

In a way analogous to the application of the optical activity of quartz by CHANDRASEKHARAN ${ }^{7}$ to discriminate between some selection rules in Raman scattering, it might be of interest to seek for the same information in linearly birefringent crystals or even in cubic crystals with sufficiently large Faraday rotations. Among the latter, the europium chalcogenides and the $\mathrm{Cd}-\mathrm{Cr}$ spinels are obvious candidates for an application of their magnetic birefringence in Ramanscattering studies.

In the field of petrography, it is hoped that this work may bring a new tool to the mineralogist, in that the light-scattering configuration now enables one to make rapid and reliable visual measurements of linear and circular birefringence on properly prepared samples.

I wish to address my most sincere thanks to Prof. G. Busch for his encouraging interest, his suggestions and his generous hospitality, which made this investigation possible. I am also grateful to Prof. F. LAves for the interest shown in this work and for stimulating suggestions. Moreover, I feel indebted to Dr. O. Vogr and Dr. E. Kaldis, who supplied the crystals, to Mr. M. LANDoLt for beneficial discussions, and to Mr. A. Heusser, whose skilful technical assistance was greatly appreciated. The financial support was provided by the Swiss National Fund for Scientific Research. 